\title{
Relevant patient characteristics for guiding tailored integrated diabetes primary care: a systematic review
}

\author{
Dorijn F.L. Hertroijs ${ }^{1}$, Arianne M.J. Elissen ${ }^{1}$, Martijn C.G.J. Brouwers ${ }^{2}$, Nicolaas C. Schaper ${ }^{3}$ and \\ Dirk Ruwaard ${ }^{1}$ \\ ${ }^{1}$ Department of Health Services Research, CAPHRI Care and Public Health Research Institute, Faculty of Health, \\ Medicine and Life Sciences, Maastricht University, Maastricht, The Netherlands \\ ${ }^{2}$ Department of Internal Medicine, Division of Endocrinology, CARIM School for Cardiovascular Diseases, Maastricht \\ University Medical Centre, Maastricht, The Netherlands \\ ${ }^{3}$ Department of Internal Medicine, Division of Endocrinology, CAPHRI School for Public Health and Primary Care, \\ Faculty of Health, Medicine and Life Sciences, Maastricht University Medical Center, Maastricht, The Netherlands
}

\begin{abstract}
Aim: To identify which patient-related effect modifiers influence the outcomes of integrated care programs for type 2 diabetes in primary care. Background: Integrated care is a widespread management strategy for the treatment of type 2 diabetes. However, most integrated care programs are not tailored to patients' needs, preferences and abilities. There is increasing consensus that such a patient-centered approach could improve the management of type 2 diabetes. Thus far, it remains unclear which patient-related effect modifiers should guide such an approach. Methods: PubMed, CINAHL and EMBASE were searched for empirical studies published after 1998. A systematic literature review was conducted according to the PRISMA guidelines. Findings: In total, 23 out of 1015 studies were included. A total of 21 studies measured the effects of integrated diabetes care programs on hemoglobin $\mathrm{A} 1 \mathrm{c}(\mathrm{HbA} 1 \mathrm{c})$ and three on low-density lipoprotein cholesterol, systolic blood pressure and health-care utilization. In total, 49 patient characteristics were assessed as potential effect modifiers with $\mathrm{HbA1c}$ as an outcome, of which 46 were person or health-related and only three were context-related. Younger age, insulin therapy and longer disease duration were associated with higher $\mathrm{HbA1c}$ levels in cross-sectional and longitudinal studies. Higher baseline $\mathrm{HbA1}$ c was associated with higher $\mathrm{HbA1c}$ at follow-up in longitudinal studies. Information on context- and person-related characteristics was limited, but is necessary to help identify the care needs of individual patients and implement an effective integrated type 2 diabetes tailored care program.
\end{abstract}

Key words: integrated health-care systems; patient-centered care; primary care; review; type 2 diabetes mellitus

Received 17 February 2017; revised 19 December 2017; accepted 21 December 2017; first published online 6 February 2018

\section{Introduction}

Diabetes is one of the most prevalent chronic conditions worldwide and a public health priority in many countries (Tamayo et al., 2014; International Diabetes Federation, 2015). In Europe, an estimated 9.8 million people suffer from diabetes; type 2 diabetes is responsible for $90 \%$ of cases. People with type 2 diabetes are at high risk for developing complications, such as cardiovascular disease and kidney failure, which in turn lead to increased health-care costs (Tamayo et al., 2014;

Correspondence to: Dorijn F.L. Hertroijs, Department of Health Services Research, CAPHRI Care and Public Health Research Institute, Faculty of Health, Medicine and Life Sciences, Maastricht University, Duboisdomein 30, 6299 GT, Maastricht, The Netherlands. Email: d.hertroijs@maastrichtuniversity.nl

(C) Cambridge University Press 2018. This is an Open Access article, distributed under the terms of the Creative Commons Attribution licence (http://creativecommons.org/licenses/by/4.0/), which permits unrestricted re-use, distribution, and reproduction in any medium, provided the original work is properly cited. 
International Diabetes Federation, 2015). To prevent diabetes-related co-morbidities and complications, and lower medical care expenditure for patients with type 2 diabetes, it is important to implement effective and efficient management strategies. An example of such a strategy is the implementation of integrated care. It aims to improve patient care and experience through improved coordination (Shaw et al., 2011).

The implementation of integrated care programs is widespread in North America, Europe, and other parts of the world (Kodner, 2009; Shaw et al., 2011). However, most integrated care programs are not tailored to patients' needs and preferences, but rather highly standardized according to evidence-based guidelines for specific diseases, such as diabetes. Findings from recent studies suggest that not all patients benefit equally from such a standardized approach (Rothe et al., 2008; Pimouguet et al., 2011; Elissen et al., 2012). These studies report that patients with poorly controlled diabetes benefit mostly from intensive, providerdriven disease management, whereas patients with adequate glucose levels might maintain these levels independent of the type of care they receive.

In 2012, the European Association for the Study of Diabetes and the American Diabetes Association recommended a more patient-centered approach for the management of type 2 diabetes (Inzucchi et al., 2012). In a patient-centered approach, care is tailored according to individual patient needs and preferences (Commitee on Quality of Health Care in America; Institute of Medicine, 2001; Inzucchi et al., 2012; American geriatrics society expert panel on person-centered care, 2016; Coulourides Kogan et al., 2016). It draws on the concept of 'mass customization', where goods and services are delivered with enough variety and customization that nearly everyone finds exactly what they want (Tseng and $\mathrm{Hu}, 2014)$. Dividing the population based on health-care needs creates groups that are more homogenous than the population as a whole. Hence, care offered to these groups will be more tailored to the patients' needs, while acknowledging that a certain amount of heterogeneity within the subgroups will remain.

There is increasing consensus that a patientcentered approach could improve the management of type 2 diabetes (Inzucchi et al., 2012).
However, to date, it is unclear what the best method is for establishing patient-centered care (Epstein and Street, 2011). Since intensive, provider-driven disease management is not beneficial to every type 2 diabetes patient, several studies have pointed toward patient characteristics - for example, number of co-morbidities, disease duration or attitude - as possible effect modifiers of treatment (Hasnain-Wynia and Baker, 2006; Inzucchi et al., 2012; Riddle and Karl, 2012; Scheen, 2016). These effect modifiers could be used to identify patients with different care needs and preferences, and subsequently serve as input to tailor treatment (Goldberger and Buxton, 2013; Constand et al., 2014) . However, it is unclear which effect modifiers should guide a more patient-centered approach. Therefore, the aim of this systematic review was to identify which patient effect modifiers influence the outcomes of integrated care programs for type 2 diabetes in primary care. These effect modifiers can help to segment the chronically ill population into subgroups with similar health-care needs for whom, based on insight into their needs and preferences, a range of matching care and support options can be developed.

This review is the first part of the research project entitled 'PROFiling patients' healthcare needs to support Integrated, person-centered models for Long-term disease management (PROFIle)' (Elissen et al., 2016). The aim of this four-year Dutch project is explicitly not to develop another disease-specific approach, but we use type 2 diabetes as starting point to develop, validate and test so-called 'patient profiles' as an instrument to support more patient-centered chronic care management in practice.

\section{Methods}

\section{Data sources and searches}

A systematic literature search according to PRISMA guidelines (Moher et al., 2009) was performed on PubMed, CINAHL and EMBASE databases in January 2015. Included were Englishor Dutch-language randomized controlled trials (RCT), prospective and retrospective cohort- and cross-sectional studies which: (1) focused on integrated care (defined below); (2) included adult patients $(\geqslant 18$ years) with type 2 diabetes; (3) were 
set in primary care; (4) measured effects on 1 or more measures of diabetes management [hemoglobin A1c (HbA1c), low-density lipoprotein cholesterol (LDL-c) and systolic blood pressure (SBP)], and/or health-care utilization as outcome variables; and (5) included sub-analyses with patient characteristics as independent variables. In line with previous research, integrated care was defined as interventions combining two or more components of the well-known Chronic Care Model (CCM) (Busetto et al., 2016). The CCM stresses the need for a more proactive health-care system by focusing on four components: selfmanagement support (eg, patient education), decision support (eg, evidence-based guidelines), delivery system design (eg, care process) and clinical information systems (eg, electronic registries)
(McCulloch et al., 1998; Coulter et al., 2015). Since the CCM was developed in 1998, only studies published in or after 1998 were included (Austin et al., 2000). The search strategy included targeted terms related to diabetes, integrated care, CCM components, care outcomes and subgroup analyses based on patient characteristics. The complete search terms and search string can be found in Table 1. The snowball method was used to search for other relevant studies.

\section{Study selection}

Potentially relevant studies were retrieved from the electronic databases based on the inclusion criteria in three screening rounds. First, titles and abstracts were screened. The first 50 titles and abstracts were screened independently by two

Table 1 Search terms and search string

\begin{tabular}{|c|c|c|}
\hline$\#$ & Category & Search terms \\
\hline 1 & Diabetes & $\begin{array}{l}\text { Diabetes OR diabetes mellitus OR diabetic patient OR type } 2 \text { diabetes OR type } 2 \text { diabetes } \\
\text { mellitus OR T2DM OR NIDDM }\end{array}$ \\
\hline 2 & Integrated care & $\begin{array}{l}\text { Integrated care OR disease management OR disease state management OR } \\
\text { comprehensive healthcare OR comprehensive health care OR shared care OR coordinated } \\
\text { care OR case management OR chronic care model OR primary care OR primary health } \\
\text { care OR outpatient clinic OR outpatient services OR primary health care OR primary } \\
\text { healthcare OR primary health clinics OR general practice OR family practice OR } \\
\text { community care }\end{array}$ \\
\hline 3 & $\begin{array}{l}\text { CCM - self-management } \\
\text { support }\end{array}$ & $\begin{array}{l}\text { Self-management OR self-management support OR self-care OR patient-centeredness } \\
\text { OR patient-centered care OR behavioral support OR motivational support OR self- } \\
\text { management education OR patient education }\end{array}$ \\
\hline 4 & $\begin{array}{l}\mathrm{CCM} \text { - delivery system } \\
\text { design }\end{array}$ & $\begin{array}{l}\text { Delivery system design OR care pathway OR critical pathway OR individualized care OR } \\
\text { clinical case management OR medicines management OR medication management OR } \\
\text { comorbidities management OR health literacy OR cultural sensitivity OR practice nurse OR } \\
\text { care team OR health care team Or healthcare team OR patient care team OR personalized } \\
\text { care OR personalized management OR individualized management OR multidisciplinary } \\
\text { care team OR tailored care OR tailored support OR multidisciplinary care }\end{array}$ \\
\hline 5 & CCM - decision support & $\begin{array}{l}\text { Decision support, clinical reminders, clinician reminders, patient reminders, provider } \\
\text { education, reminder systems, individualized care plans, individual care plans }\end{array}$ \\
\hline 6 & $\begin{array}{l}\mathrm{CCM} \text { - clinical } \\
\text { information system }\end{array}$ & $\begin{array}{l}\text { Clinical information system, clinical information systems, clinical registry, health } \\
\text { information system, health information systems, health information technology, } \\
\text { electronic registry, clinical reminders, clinician reminders, patients reminders, provider } \\
\text { feedback, performance monitoring, ICT device, patient portal, patient registry, diabetes } \\
\text { registry, telemonitoring, telehealth, teleassistance, telehomecare, videoconferencing, } \\
\text { mobile phone }\end{array}$ \\
\hline 7 & Outcome measures & $\begin{array}{l}\text { Glycemic control, glycaemic control, diabetic control, diabetes control, diabetes status, } \\
\text { Charlson Comborbidity Index, resource use, health care use, health care utility, service } \\
\text { use, resource utility, service utility }\end{array}$ \\
\hline 8 & Subgroup analysis & $\begin{array}{l}\text { Factor, predictor, predictive factor, determinant, patient characteristic, patient } \\
\text { characteristics, patient feature, patient features, patient dynamics, subgroup, subgroups, } \\
\text { segment, strata, classes }\end{array}$ \\
\hline 9 & Complete search string & $\begin{array}{l}\# 1 \text { AND (\#2 OR (\#3 AND \#4) OR (\#3 AND \#5) OR (\#3 AND \#6) OR (\#4 AND \#5) OR (\#4 AND \#6) } \\
\text { OR (\#5 AND \#6)) AND \#7 AND \#8 }\end{array}$ \\
\hline
\end{tabular}

$\mathrm{CCM}=$ Chronic Care Model. 
reviewers (D.H. and A.E.). More than 90\% agreement was reached. Therefore, the remainder of the titles and abstracts were screened by 1 reviewer (D.H.). Second, the first 20 full texts were screened independently by two reviewers (D.H. and A.E.). Again, more than $90 \%$ agreement was reached and therefore, each reviewer independently screened half of the full texts. Third, the reference lists of the included studies were screened to obtain additional studies. Steps 1 and 2 of the study selection process were then repeated.

\section{Data extraction and quality assessment}

Descriptive data on studies were extracted by 1 reviewer (D.H.) between August and October 2015. Studies were coded for author names, year of publication, country, study design, length of follow-up, population size, age, percentage of males and CCM components. In case of uncertainties, a group discussion was held with two other authors (A.E. and M.B.).

The Effective Public Health Practice Project Quality Assessment Tool (EPHPP) was used to assess the quality of the included studies (ArmijoOlivo et al., 2012). This tool was chosen because it allows the assessment of different study designs. The studies were rated based on six domains: (1) selection bias; (2) study design; (3) confounders; (4) blinding; (5) data collection; and (6) withdrawals and dropouts. Each domain was rated as 'strong,' 'moderate' or 'weak'. A global rating was given based on the number of weak components.

Two reviewers (D.H. and M.B.) independently performed the quality assessment for each study. Disagreements were resolved via discussion conform EPHPP guidelines.

\section{Data synthesis and analysis}

The included studies were categorized according to: (1) the reported outcome(s) of interest (HbA1c, LDL-c, SBP and/or health-care utilization); and (2) the type of patient characteristic(s) investigated in subgroup analyses. Characteristics were classified as person-related (predisposing), context-related (enabling) or health-related (illness level) characteristics according to Andersen and Newman's (1973) Behavioral Model of Health Service Use. The model provides a theoretical framework for viewing health services utilization, taking into account both societal and individual characteristics.
The model was chosen, because the individual characteristics can inform tailored care by, for example, helping determine the best intensity of care for the individual patient. Relationships between outcomes and characteristics were depicted as ' + ' for significant positive relationships, as ' - 'for significant negative relationships and as 'o' for non-significant relationships.

\section{Results}

\section{Search results}

In total, 1374 studies were identified through electronic databases and by checking the references of the included studies. Figure 1 shows the flow diagram of the study selection. Most studies were excluded because none relevant outcomes were reported $(n=453)$, and/or type of care was not integrated $(n=257)$. After the title, abstract and full text screening, 27 studies were included (Groeneveld et al., 2001; Ostgren et al., 2002; El-Kebbi et al., 2003; Rothman et al., 2003; Rothman et al., 2004; Uitewaal et al., 2004; Benoit et al., 2005; Sperl-Hillen and O'Connor, 2005; Uitewaal et al., 2005; De Alba Garcia et al., 2006; Nielsen et al., 2006; Taweepolcharoen et al., 2006; Trief et al., 2006; Wahba and Chang, 2007; Mold et al., 2008; Al Omari et al., 2009; De Fine Olivarius et al., 2009; Robinson et al., 2009; Kellow et al., 2011; Cardenas-Valladolid et al., 2012; Elissen et al., 2012; Liu et al., 2013; Quah et al., 2013; LeBlanc et al., 2015; Luijks et al., 2015; Moreira et al., 2015; Quinn et al., 2016).

\section{Quality assessment}

The methodological quality of the included studies can be found in Supplementary Table 1 . The domains with the most 'weak' ratings were confounders $(n=10)$, blinding $(n=9)$ and selection bias $(n=9)$. Almost all studies $(n=25)$ scored high on the domain data collection. The overall study quality was strong for four studies, moderate for 11 studies and low for 12 studies. Most studies with low quality had a cross-sectional study design and did not report on or adjust for possible confounders.

\section{Study and sample characteristics}

Of the included studies, nine $(33.3 \%)$ were retrospective cohort studies, seven (25.9\%) 


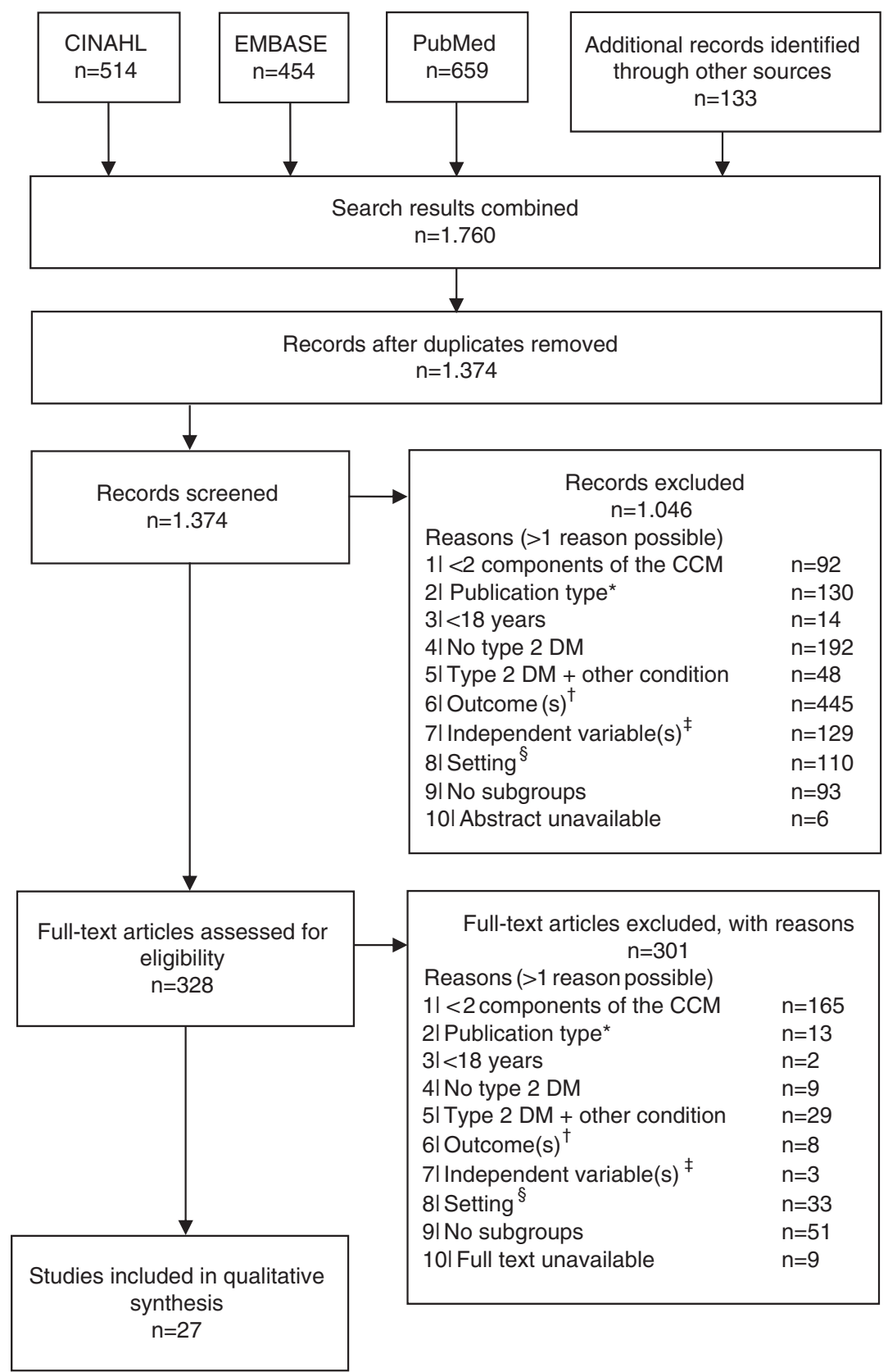

Figure 1 Flow diagram of the study selection. *Qualitative, or mixed-method studies; ${ }^{\dagger}$ any outcome other than hemoglobin A1c, low-density lipoprotein cholesterol, blood pressure or health-care utilization; ${ }^{*}$ independent variable is not a person-, context- or health-related patient characteristic (eg, health-care provider characteristics); ${ }^{\S}$ setting is not a primary care setting (eg, hospital). CCM $=$ Chronic Care Model; DM=diabetes mellitus. 
cross-sectional studies, seven (25.9\%) (randomized) controlled studies and four (14.8\%) prospective cohort studies. Table 2 shows that the median follow-up duration for retrospective cohort, prospective cohort and randomized controlled studies $(n=20)$ was 15 months (range 6-112). The median sample size consisted of 376 individuals (range 80-105056) with an average age of 60.0 years (range 50.5-70.9); the percentage of male subjects ranged from 31.3 to 68.0 .

Table 2 also provides an overview of the CCM components implemented in each study. Eight studies included all four components of the CCM model. The CCM component delivery system design was included in most studies $(n=25)$, followed by self-management support $(n=20)$. Of the studies that included the components delivery system design, most introduced a care team $(n=13)$, followed by regular follow-up visits $(n=8)$. Self-management support was mostly realized through individual educational sessions on diabetes, health and nutrition $(n=14)$.

\section{Outcome variables}

\section{$H b A 1 c$}

In total, 18 uncontrolled studies - including prospective, retrospective and cross-sectional cohort designs - measured the effects of integrated care programs on HbA1c. In addition, seven studies compared the influence of patient characteristics on the effectiveness of integrated diabetes care programs between intervention and control groups. In total, 51 patient characteristics were assessed as potential effect modifiers of the relationship between integrated care and HbA1c. The results will be presented according to study design. For RCTs all characteristics assessed by this study design will be discussed. Due to the high number of characteristics assessed by the crosssectional, retrospective and prospective cohort studies, only characteristics assessed by three or more studies will be presented.

(Randomized) controlled trials: Five RCTs and two controlled trials (CTs) compared the influence of patient characteristics on the effectiveness of integrated diabetes care programs on the HbA1c level between intervention and control groups (Table 3). In total, eight patient characteristics were evaluated as potential modifiers.
Sex and age were the person-related characteristics evaluated as potential effect modifiers. Three studies assessed sex as a potential modifier, of which two found that women in the intervention group had statistically significant lower HbA1c values at follow-up compared to women in the control group (Uitewaal et al., 2005; Nielsen et al., 2006). For men, no statistically significant difference was found. The third study did not find a statistically significant relationship (Moreira et al., 2015). Age was assessed by two studies. Both found that younger patients receiving integrated diabetes care had statistically significantly lower HbA1c values at follow-up compared to patients receiving usual care (Moreira et al., 2015; Quinn et al., 2016).

Three health-related characteristics were evaluated as potential effect modifiers of the relationship between integrated diabetes care programs and HbA1c: literacy status, income and number schooling years. Literacy status was assessed by one study (Rothman et al., 2004), which found that patients in the intervention group with low literacy status ( $\leqslant 6$ th grade) had statistically significant lower HbA1c values at follow-up compared to patients with low literacy status receiving usual care. Monthly income and number of schooling years were also each assessed by one study. Patients with lower monthly income $(\leqslant \$ 118.26)$ and $\leqslant$ four years of schooling at baseline receiving integrated diabetes care had significantly lower HbA1c values at follow-up compared to patient receiving usual care (Moreira et al., 2015).

Three health-related characteristics were evaluated as potential effect modifiers of the relationship between integrated diabetes care programs and $\mathrm{HbA1c}$ : fasting blood glucose (FBG), depression and diabetes mellitus (DM) duration. Each characteristic was assessed by one study. Patients with high FBG $(>10 \mathrm{mmol} / \mathrm{L})$ at baseline receiving integrated diabetes care had significantly lower HbA1c levels at follow-up compared to patients receiving usual care (Groeneveld et al., 2001). For patients with a FBG $\leqslant 10 \mathrm{mmol} / \mathrm{L}$ no significant difference was found in HbA1c levels at follow-up between the intervention and control groups. Depression was not an effect modifier of the association between integrated diabetes care programs and HbA1c (Trief et al., 2006). Patients with a DM duration < five years receiving integrated diabetes care had significantly lower HbA1c levels 
Table 2 Study and sample characteristics

\begin{tabular}{|c|c|c|c|c|c|c|c|c|c|c|}
\hline \multicolumn{4}{|l|}{ Study characteristics } & \multicolumn{3}{|c|}{ Sample characteristics } & \multicolumn{4}{|l|}{$\mathrm{CCM}$} \\
\hline \multirow[b]{2}{*}{ Study } & \multirow[b]{2}{*}{ Country } & \multirow[b]{2}{*}{$\begin{array}{l}\text { Study } \\
\text { design }\end{array}$} & \multirow[b]{2}{*}{$\begin{array}{l}\text { Follow- } \\
\text { up } \\
\text { (months) }\end{array}$} & \multirow[b]{2}{*}{$n$} & \multirow[b]{2}{*}{$\begin{array}{l}\text { Age (SD or } \\
\text { range) }\end{array}$} & \multirow[b]{2}{*}{$\begin{array}{l}\text { Sex } \\
\text { (\% male) }\end{array}$} & \multicolumn{4}{|l|}{ Description of components } \\
\hline & & & & & & & $\begin{array}{l}\text { Self-management } \\
\text { support }\end{array}$ & Delivery system design & $\begin{array}{l}\text { Clinical information } \\
\text { systems }\end{array}$ & Decision support \\
\hline $\begin{array}{l}\text { Al Omari et al. } \\
\text { (2009) }\end{array}$ & JOR & $\mathrm{CS}$ & $\mathrm{N} / \mathrm{A}$ & 337 & $54.1(11.3)$ & 52.1 & $\begin{array}{l}\text { Regular group } \\
\text { counseling with the } \\
\text { presence of family } \\
\text { physicians, nurses, } \\
\text { pharmacists and } \\
\text { dieticians } \\
\text { Leaflets related to } \\
\text { diabetes }\end{array}$ & $\begin{array}{l}\text { Care team (doctor and } \\
\text { diabetic nurse) } \\
\text { Regular follow-up: patient } \\
\text { has to see the physician to } \\
\text { take the prescription on a } \\
\text { monthly basis }\end{array}$ & & \\
\hline Benoit et al. (2005) & USA & $\mathrm{RC}$ & 24 & 573 & $55.4(10.1)$ & 31.3 & & $\begin{array}{l}\text { The nurse educator is the } \\
\text { case manager } \\
\text { Nurse educator identifies } \\
\text { individual service and } \\
\text { access needs of patients } \\
\text { Nurse communicates with } \\
\text { the primary care } \\
\text { physician regarding } \\
\text { clinical issues }\end{array}$ & $\begin{array}{l}\text { Nurse educator } \\
\text { follows up on } \\
\text { missed patient } \\
\text { appointments } \\
\text { Diabetes electronic } \\
\text { medical system } \\
\text { software }\end{array}$ & \\
\hline $\begin{array}{l}\text { Cardenas- } \\
\text { Valladolid et al. } \\
\text { (2012) }\end{array}$ & ES & PC & 24 & 23488 & $69.7(14.5)$ & 48.4 & $\begin{array}{l}\text { Interventions focused } \\
\text { on drug therapy } \\
\text { compliance, change in } \\
\text { lifestyle, health } \\
\text { education and self- } \\
\text { management }\end{array}$ & & $\begin{array}{l}\text { Computerized clinical } \\
\text { record }\end{array}$ & \\
\hline $\begin{array}{l}\text { De Fine Olivarius } \\
\text { et al. (2009) }\end{array}$ & DK & $\mathrm{PC}$ & 66 & 581 & $64.7(55.7-73.2)$ & 51.9 & $\begin{array}{l}\text { Individualized goal } \\
\text { setting }\end{array}$ & $\begin{array}{l}\text { Follow-up every } 3 \text { months } \\
\text { annual screening } \\
\text { for diabetic complications }\end{array}$ & $\begin{array}{l}\text { Annual descriptive } \\
\text { feedback reports on } \\
\text { individual patients }\end{array}$ & $\begin{array}{l}\text { Clinical guidelines } \\
\text { supported by annual } \\
\text { half day seminar }\end{array}$ \\
\hline $\begin{array}{l}\text { Elissen et al. } \\
\text { (2012) }\end{array}$ & NL & $\mathrm{RC}$ & $20-24$ & 105056 & $65.7(11.9)$ & Unknown & $\begin{array}{l}\text { National Diabetes Care } \\
\text { Standard includes } \\
\text { general modules on } \\
\text { information, } \\
\text { education and self- } \\
\text { management support, } \\
\text { smoking, cessation, } \\
\text { physical activity, } \\
\text { nutrition and diet }\end{array}$ & $\begin{array}{l}\text { Care team (GP, practice } \\
\text { nurse) }\end{array}$ & $\begin{array}{l}\text { Shared diabetes } \\
\text { patient registry }\end{array}$ & $\begin{array}{l}\text { Defined frequency of } \\
\text { GP visits, regular } \\
\text { foot and eye } \\
\text { examinations, } \\
\text { laboratory testing }\end{array}$ \\
\hline $\begin{array}{l}\text { El-Kebbi et al. } \\
(2003)\end{array}$ & USA & $\mathrm{RC}$ & $5-12$ & 2539 & $55.0(12.0)$ & 44.0 & $\begin{array}{l}\text { Education program } \\
\text { emphasizing lifestyle } \\
\text { modifications and } \\
\text { self-management } \\
\text { skills offered to all } \\
\text { patients at their initial } \\
\text { visit and projects } 6 \text { to } 8 \\
\text { return visits within the } \\
\text { first year }\end{array}$ & $\begin{array}{l}\text { Patients cared for by a team } \\
\text { of nurse providers, } \\
\text { physicians, dietitians, } \\
\text { podiatrists and a social } \\
\text { worker }\end{array}$ & & $\begin{array}{l}\text { If glycemic goals are } \\
\text { not met after the first } \\
\text { one to two months, } \\
\text { pharmacologic } \\
\text { therapy is started or } \\
\text { advanced according } \\
\text { to a stepped-care } \\
\text { protocol for } \\
\text { intensification of } \\
\text { therapy }\end{array}$ \\
\hline
\end{tabular}


Table 2 (Continued)

\begin{tabular}{|c|c|c|c|c|c|c|c|c|c|c|}
\hline \multicolumn{4}{|c|}{ Study characteristics } & \multicolumn{3}{|c|}{ Sample characteristics } & \multicolumn{4}{|l|}{$\mathrm{CCM}$} \\
\hline \multirow[b]{2}{*}{ Study } & \multirow[b]{2}{*}{ Country } & \multirow[b]{2}{*}{$\begin{array}{l}\text { Study } \\
\text { design }\end{array}$} & \multirow[b]{2}{*}{$\begin{array}{l}\text { Follow- } \\
\text { up } \\
\text { (months) }\end{array}$} & \multirow[b]{2}{*}{$n$} & \multirow[b]{2}{*}{$\begin{array}{l}\text { Age (SD or } \\
\text { range) }\end{array}$} & \multirow[b]{2}{*}{$\begin{array}{l}\text { Sex } \\
(\% \text { male })\end{array}$} & \multicolumn{4}{|l|}{ Description of components } \\
\hline & & & & & & & $\begin{array}{l}\text { Self-management } \\
\text { support }\end{array}$ & Delivery system design & $\begin{array}{l}\text { Clinical information } \\
\text { systems }\end{array}$ & Decision support \\
\hline $\begin{array}{l}\text { De Alba Garcia } \\
\text { et al. (2006) }\end{array}$ & Mex & CS & $\mathrm{N} / \mathrm{A}$ & 796 & $60.5(10.8)$ & 38.6 & $\begin{array}{l}\text { Diabetes and nutrition } \\
\text { education } \\
\text { Diabetes and exercise } \\
\text { support groups }\end{array}$ & $\begin{array}{l}\text { Care team (physicians, } \\
\text { nutritionist and } \\
\text { psychologist) }\end{array}$ & & \\
\hline $\begin{array}{l}\text { Groeneveld et al. } \\
\text { (2001) }\end{array}$ & $\mathrm{NL}$ & $\mathrm{RCT}$ & 12 & $\begin{array}{l}\text { I: } 91 \\
\text { C: } 155\end{array}$ & $\begin{array}{r}\text { I: } 62.7(11) \\
\text { C: } 62.3(10)\end{array}$ & $\begin{array}{r}\text { I: } 34.1 \\
\text { C: } 46.4\end{array}$ & $\begin{array}{l}\text { Counseling by a } \\
\text { diabetes educator } \\
\text { (nurse) and dietician at } \\
\text { the 'Diabetes Service', } \\
\text { a monitoring and } \\
\text { advisory service }\end{array}$ & $\begin{array}{l}\text { Care team consisting of } \\
\text { diabetes educator (nurse), } \\
\text { dietician and GP } \\
\text { Patients were called up and } \\
\text { reviewed every three } \\
\text { months. If insulin was } \\
\text { started contacts were } \\
\text { more frequent }\end{array}$ & & $\begin{array}{l}\text { GP responsible for } \\
\text { implementation of } \\
\text { therapeutic advice of } \\
\text { the Diabetes Service }\end{array}$ \\
\hline $\begin{array}{l}\text { Kellow, Savige } \\
\text { and Khalil (2011) }\end{array}$ & AUS & $\mathrm{RC}$ & 60 & 272 & $62.1(11.6)$ & 49.0 & $\begin{array}{l}\text { Diabetes education at } \\
\text { the health service } \\
\text { diabetes education } \\
\text { department }\end{array}$ & $\begin{array}{l}\text { Care team (GP, diabetes } \\
\text { educator). Diabetes } \\
\text { educator referred patients } \\
\text { for additional optometry, } \\
\text { podiatry and dietetic } \\
\text { appointments as required }\end{array}$ & & \\
\hline $\begin{array}{l}\text { LeBlanc et al. } \\
\text { (2015) }\end{array}$ & USA & $\mathrm{RC}$ & 12 & 14430 & $63(55.0-76.0)$ & 52.5 & & & $\begin{array}{l}\text { Electronic medical } \\
\text { record system }\end{array}$ & $\begin{array}{l}\text { Evidence-based } \\
\text { treatment guidelines }\end{array}$ \\
\hline Liu et al. (2013) & $\mathrm{CH}$ & CS & $\mathrm{N} / \mathrm{A}$ & 960 & $68.3(10.4)$ & 39.6 & & $\begin{array}{l}\text { Health management } \\
\text { Follow-up every three } \\
\text { months }\end{array}$ & & $\begin{array}{l}\text { Community diabetes } \\
\text { prevention and } \\
\text { treatment guidelines } \\
\text { provide glycemic } \\
\text { control targets }\end{array}$ \\
\hline Luijks et al. (2015) & $\mathrm{NL}$ & $\mathrm{PC}$ & 60 & 610 & $63(12.5)$ & 48.2 & & $\begin{array}{l}\text { Routine three-monthly } \\
\text { check-up visits }\end{array}$ & $\begin{array}{l}\text { Electronic medical } \\
\text { record system }\end{array}$ & \\
\hline Mold et al. (2008) & UK & $\mathrm{RC}$ & 11 & 646 & $\begin{array}{l}<50: 16.4 \% \\
50-59: 18.3 \% \\
60-69: 31.1 \% \\
\geqslant 70: 34.2 \%\end{array}$ & 54.3 & $\begin{array}{l}\text { Dietary advice is } \\
\text { offered at each } \\
\text { consultation }\end{array}$ & $\begin{array}{l}\text { Care team (GP, practice } \\
\text { nurse) } \\
\text { Patients initially see the GP } \\
\text { and are then referred to } \\
\text { the practice nurse }\end{array}$ & $\begin{array}{l}\text { Electronic medical } \\
\text { record system }\end{array}$ & \\
\hline $\begin{array}{l}\text { Moreira et al. } \\
(2015)\end{array}$ & Brazil & $\mathrm{RCT}$ & 12 & $\begin{array}{l}\text { I: } 40 \\
\text { C: } 40\end{array}$ & $\begin{array}{l}\text { I: } 50.0(6.5) \\
\text { C: } 50.3(7.3)\end{array}$ & $\begin{array}{l}\text { I: } 40 \\
\text { C: } 30\end{array}$ & $\begin{array}{l}\text { Educational activities } \\
\text { focused on providing } \\
\text { orientation about } \\
\text { physical activities, } \\
\text { healthy diet, } \\
\text { monitoring capillary } \\
\text { glycemia, and acute } \\
\text { and chronic } \\
\text { complications }\end{array}$ & $\begin{array}{l}\text { Quarterly nursing } \\
\text { consultations, bimonthly } \\
\text { educational group } \\
\text { activities. When } \\
\text { necessary referral for a } \\
\text { consultation with a } \\
\text { primary health-care } \\
\text { physician, nurse, } \\
\text { nephrologist, pharmacist } \\
\text { and nutritionist. Home } \\
\text { visits and phone contacts } \\
\text { on a monthly basis with } \\
\text { the case manager }\end{array}$ & & \\
\hline
\end{tabular}


Table 2 (Continued)

\begin{tabular}{|c|c|c|c|c|c|c|c|c|c|c|}
\hline \multicolumn{4}{|l|}{ Study characteristics } & \multicolumn{3}{|c|}{ Sample characteristics } & \multicolumn{4}{|l|}{$\mathrm{CCM}$} \\
\hline \multirow[b]{2}{*}{ Study } & \multirow[b]{2}{*}{ Country } & \multirow[b]{2}{*}{$\begin{array}{l}\text { Study } \\
\text { design }\end{array}$} & \multirow[b]{2}{*}{$\begin{array}{l}\text { Follow- } \\
\text { up } \\
\text { (months) }\end{array}$} & \multirow[b]{2}{*}{$n$} & \multirow[b]{2}{*}{$\begin{array}{l}\text { Age (SD or } \\
\text { range) }\end{array}$} & \multirow[b]{2}{*}{$\begin{array}{l}\text { Sex } \\
\text { (\% male) }\end{array}$} & \multicolumn{4}{|c|}{ Description of components } \\
\hline & & & & & & & $\begin{array}{l}\text { Self-management } \\
\text { support }\end{array}$ & Delivery system design & $\begin{array}{l}\text { Clinical information } \\
\text { systems }\end{array}$ & Decision support \\
\hline $\begin{array}{l}\text { Nielsen et al. } \\
(2006)\end{array}$ & DK & $\mathrm{RCT}$ & 72 & $\begin{array}{l}\text { I: } 459 \\
\text { C: } 415\end{array}$ & $\begin{array}{l}\text { Median } \\
\text { I: } 63.0 \\
(53.8-71.4) \\
\text { C: } 63.7 \\
(65.6-71.6)\end{array}$ & $\begin{array}{l}\text { I: } 48.8 \\
\text { C: } 52.3\end{array}$ & $\begin{array}{l}\text { Individualized goal } \\
\text { setting }\end{array}$ & $\begin{array}{l}\text { Follow-up every three } \\
\text { months } \\
\text { Annual screening for } \\
\text { diabetic complications }\end{array}$ & $\begin{array}{l}\text { Annual descriptive } \\
\text { feedback reports on } \\
\text { individual patients }\end{array}$ & $\begin{array}{l}\text { Clinical guidelines } \\
\text { supported by annual } \\
\text { half day seminar }\end{array}$ \\
\hline $\begin{array}{l}\text { Óstgren et al. } \\
\text { (2002) }\end{array}$ & SWE & CS & $\mathrm{N} / \mathrm{A}$ & 376 & $\begin{array}{c}\text { HbA1c <6.5: } \\
69.6(10.4) \\
\text { HbA1c } \geqslant 6.5: \\
70.9(9.8)\end{array}$ & 50.5 & $\begin{array}{l}\text { Structured education } \\
\text { program }\end{array}$ & $\begin{array}{l}\text { Specially trained nurses, } \\
\text { supervised by the } \\
\text { physician. Team also } \\
\text { included a dietician and a } \\
\text { podiatrist }\end{array}$ & & $\begin{array}{l}\text { Structured treatment } \\
\text { program, including } \\
\text { annual check-up at } \\
\text { hypertension and } \\
\text { diabetes outpatient } \\
\text { clinic including } \\
\text { examinations } \\
\text { concerning vision, } \\
\text { peripheral sensibility } \\
\text { of vibration and } \\
\text { peripheral pulsation } \\
\text { and laboratory tests }\end{array}$ \\
\hline Quah et al. (2013) & SG & CS & $\mathrm{N} / \mathrm{A}$ & 688 & $62.2(11.1)$ & 44.0 & & $\begin{array}{l}\text { Routine three-monthly visit } \\
\text { to polyclinics }\end{array}$ & Diabetes database & \\
\hline Quinn et al. (2016) & USA & $\mathrm{RCT}$ & 12 & 118 & $\begin{array}{l}\text { Age }<55 \\
\text { years: } \\
\text { I: } 47.3(6.8) \\
\text { C: } 47.5(7.5) \\
\text { Age } \geqslant 55 \\
\text { years: } \\
\text { I: } 59.0(2.9) \\
\text { C: } 59.5(2.8)\end{array}$ & $\begin{array}{l}\text { Age }<55 \\
\text { years: } \\
\text { I: } 37.3 \\
\text { C: } 62.1 \\
\text { Age } \geqslant 55 \\
\text { years: } \\
\text { I: } 68.0 \\
\text { C: } 37.0\end{array}$ & $\begin{array}{l}\text { Mobile diabetes } \\
\text { management } \\
\text { software application, } \\
\text { which allowed patient } \\
\text { to enter diabetes self- } \\
\text { care data on a phone } \\
\text { and receive } \\
\text { automated, real-time } \\
\text { messages that were } \\
\text { educational, } \\
\text { behavioral, } \\
\text { motivational and } \\
\text { specific to the entered } \\
\text { data } \\
\text { Electronic diabetes self- } \\
\text { care action plan }\end{array}$ & $\begin{array}{l}\text { Patients could } \\
\text { communicate with } \\
\text { 'virtual' case managers on } \\
\text { the phone or } \\
\text { electronically }\end{array}$ & $\begin{array}{l}\text { Quarterly online } \\
\text { reports that } \\
\text { summarized } \\
\text { patients' glycemic } \\
\text { and metabolic } \\
\text { control, etc. }\end{array}$ & Clinical guidelines \\
\hline $\begin{array}{l}\text { Robinson et al. } \\
\text { (2009) }\end{array}$ & USA & PC & 18 & 315 & $64.4(15.8)$ & 41.9 & $\begin{array}{l}\text { Self-monitoring of } \\
\text { blood glucose, foot } \\
\text { care, diet and exercise } \\
\text { modification, diabetes } \\
\text { education resources, } \\
\text { and participation in } \\
\text { planned visits, were } \\
\text { addressed through } \\
\text { individual and small } \\
\text { group appointments } \\
\text { with members of the } \\
\text { care team and through }\end{array}$ & $\begin{array}{l}\text { Care team consisting of } \\
\text { medicine resident, nurse } \\
\text { practitioner students and } \\
\text { pharmacy students } \\
\text { All participated in chronic } \\
\text { illness curriculum } \\
\text { Patients seen in individual } \\
30 \text {-minute appointments } \\
\text { by one or more of the } \\
\text { team members } \\
\text { Follow-up appointments } \\
\text { were scheduled }\end{array}$ & $\begin{array}{l}\text { An electronic clinical } \\
\text { information system } \\
\text { supplied clinical data }\end{array}$ & $\begin{array}{l}\text { Care team } \\
\text { participates in 60- } \\
\text { minute didactic } \\
\text { presentation, 30- } \\
\text { minute clinical } \\
\text { discussion session } \\
\text { focusing on patient } \\
\text { management and } \\
\text { quality improvement } \\
\text { Weekly presentation } \\
\text { topic covered }\end{array}$ \\
\hline
\end{tabular}


Table 2 (Continued)

\begin{tabular}{|c|c|c|c|c|c|c|c|c|c|c|}
\hline \multicolumn{4}{|c|}{ Study characteristics } & \multicolumn{3}{|c|}{ Sample characteristics } & \multicolumn{4}{|l|}{$\mathrm{CCM}$} \\
\hline & & & & & & & Description of componer & & & \\
\hline \multirow[t]{2}{*}{ Study } & Country & $\begin{array}{l}\text { Study } \\
\text { design }\end{array}$ & $\begin{array}{l}\text { Follow- } \\
\text { up } \\
\text { (months) }\end{array}$ & $n$ & $\begin{array}{l}\text { Age (SD or } \\
\text { range) }\end{array}$ & $\begin{array}{l}\text { Sex } \\
(\% \text { male })\end{array}$ & $\begin{array}{l}\text { Self-management } \\
\text { support }\end{array}$ & Delivery system design & $\begin{array}{l}\text { Clinical information } \\
\text { systems }\end{array}$ & Decision support \\
\hline & & & & & & & $\begin{array}{l}\text { population-based } \\
\text { quality improvement } \\
\text { projects } \\
\text { All patients in the } \\
\text { intervention group } \\
\text { were targeted for } \\
\text { individual coaching in } \\
\text { self-management } \\
\text { activities by the NP or } \\
\text { pharmacy student }\end{array}$ & & & $\begin{array}{l}\text { various aspects of } \\
\text { diabetes care }\end{array}$ \\
\hline $\begin{array}{l}\text { Rothman et al. } \\
\text { (2003) }\end{array}$ & USA & $\mathrm{RC}$ & 6 & 138 & $57.0(23-87)$ & 41.0 & $\begin{array}{l}\text { Diabetes education: } \\
\text { 1-h educational } \\
\text { session }\end{array}$ & $\begin{array}{l}\text { Three pharmacists } \\
\text { participated in the } \\
\text { program. Referrals for } \\
\text { ophthalmology, nutrition } \\
\text { and podiatry also were } \\
\text { suggested to the patient } \\
\text { and provided when } \\
\text { appropriate } \\
\text { All recommendations } \\
\text { discussed with primary } \\
\text { care provider }\end{array}$ & $\begin{array}{l}\text { Computer database } \\
\text { Patients were } \\
\text { contacted } \\
\text { approximately every } \\
2 \text { weeks through } \\
\text { phone calls, letters } \\
\text { or pharmacy visits }\end{array}$ & $\begin{array}{l}\text { Algorithms for } \\
\text { titrating insulin and } \\
\text { metformin }\end{array}$ \\
\hline $\begin{array}{l}\text { Rothman et al. } \\
\text { (2004) }\end{array}$ & USA & $\mathrm{RCT}$ & 12 & $\begin{array}{l}\text { I: } 98 \\
\text { C: } 95\end{array}$ & $\begin{array}{l}\text { I low literacy: } \\
57 \text { (10.5) } \\
\text { I high literacy: } \\
51 \text { (13.1) } \\
\text { C low literacy: } \\
59 \text { (10.4) } \\
\text { C high literacy: } \\
56 \text { (10.9) }\end{array}$ & $\begin{array}{l}\text { I low literacy: } \\
45 \\
\text { I high literacy: } \\
35 \\
\text { C low literacy: } \\
47 \\
\text { C high literacy: } \\
42\end{array}$ & $\begin{array}{l}\text { One-to-one educational } \\
\text { sessions including } \\
\text { counseling and } \\
\text { medication } \\
\text { management } \\
\text { Communication } \\
\text { individualized } \\
\text { depending on patients } \\
\text { literacy status }\end{array}$ & $\begin{array}{l}\text { Intensive diabetes } \\
\text { management from three } \\
\text { clinical pharmacist } \\
\text { practitioners and a } \\
\text { diabetes care coordinator } \\
\text { (DCC) }\end{array}$ & $\begin{array}{l}\text { Patients contacted } \\
\text { every two to four } \\
\text { weeks by telephone } \\
\text { or in person by } \\
\text { pharmacist or DCC }\end{array}$ & $\begin{array}{l}\text { Application of } \\
\text { evidence-based } \\
\text { treatment } \\
\text { algorithms to help } \\
\text { manage glucose and } \\
\text { cardiovascular risk }\end{array}$ \\
\hline $\begin{array}{l}\text { Sperl-Hillen and } \\
\text { O'Connor (2005) }\end{array}$ & USA & $\mathrm{RC}$ & 112 & $\begin{array}{l}5610- \\
7650\end{array}$ & $59-61$ & $52-54$ & $\begin{array}{l}\text { Nurses provided } \\
\text { diabetes education } \\
\text { and self-management } \\
\text { training }\end{array}$ & $\begin{array}{l}\text { Diabetes education nurses } \\
\text { work closely with primary } \\
\text { care physicians }\end{array}$ & $\begin{array}{l}\text { Patient registry. } \\
\text { Nurses use the } \\
\text { registries to guide } \\
\text { 'active outreach' to } \\
\text { high-risk patients not } \\
\text { in metabolic control } \\
\text { or missing } \\
\text { recommended tests }\end{array}$ & $\begin{array}{l}\text { Drug formulary } \\
\text { facilitated use of } \\
\text { sulfonylureas, } \\
\text { metformin, insulin, } \\
\text { fibrates and 3- } \\
\text { hydroxy-3-methyl- } \\
\text { glutaryl-coenzyme A } \\
\text { reductase inhibitors }\end{array}$ \\
\hline
\end{tabular}


Table 2 (Continued)

\begin{tabular}{|c|c|c|c|c|c|c|c|c|c|c|}
\hline \multicolumn{4}{|c|}{ Study characteristics } & \multicolumn{3}{|c|}{ Sample characteristics } & \multicolumn{4}{|l|}{$\mathrm{CCM}$} \\
\hline \multirow[b]{2}{*}{ Study } & \multirow[b]{2}{*}{ Country } & \multirow[b]{2}{*}{$\begin{array}{l}\text { Study } \\
\text { design }\end{array}$} & \multirow[b]{2}{*}{$\begin{array}{l}\text { Follow- } \\
\text { up } \\
\text { (months) }\end{array}$} & \multirow[b]{2}{*}{$n$} & \multirow[b]{2}{*}{$\begin{array}{l}\text { Age (SD or } \\
\text { range) }\end{array}$} & \multirow[b]{2}{*}{$\begin{array}{l}\text { Sex } \\
\text { (\% male) }\end{array}$} & \multicolumn{4}{|c|}{ Description of components } \\
\hline & & & & & & & $\begin{array}{l}\text { Self-management } \\
\text { support }\end{array}$ & Delivery system design & $\begin{array}{l}\text { Clinical information } \\
\text { systems }\end{array}$ & Decision support \\
\hline Trief et al. (2006) & USA & $\mathrm{CT}$ & 12 & 1665 & $70.8(6.6)$ & 37.2 & $\begin{array}{l}\text { Nurse case manager } \\
\text { provided diabetes } \\
\text { education }\end{array}$ & $\begin{array}{l}\text { Nurse case manager } \\
\text { provides, under the } \\
\text { supervision of an } \\
\text { endocrinologist, } \\
\text { treatment planning and } \\
\text { consultation to PCPs who } \\
\text { maintained decision } \\
\text { authority for their patients } \\
\text { A separate team of trained } \\
\text { research nurses conducts } \\
\text { physical and } \\
\text { psychological } \\
\text { assessments at baseline } \\
\text { and one-year follow-up }\end{array}$ & $\begin{array}{l}\text { Intervention subjects } \\
\text { received a home } \\
\text { telemedicine unit, ie, } \\
\text { a web-enabled } \\
\text { computer used to } \\
\text { upload blood } \\
\text { pressure and blood } \\
\text { glucose } \\
\text { measurements, to } \\
\text { videoconference } \\
\text { with a nurse case } \\
\text { manager and } \\
\text { dietician, and to } \\
\text { access } \\
\text { individualized } \\
\text { graphic data } \\
\text { displays and } \\
\text { educational } \\
\text { materials }\end{array}$ & \\
\hline $\begin{array}{l}\text { Uitewaal et al. } \\
(2004)\end{array}$ & $\mathrm{NL}$ & $\mathrm{RC}$ & 24 & $\begin{array}{l}\text { T: } 106 \\
\text { D: } 90\end{array}$ & $\begin{array}{l}\text { T: } 50.5(7.5) \\
\text { D: } 55.3(8.2)\end{array}$ & $\begin{array}{l}\text { T: } 43.3 \\
\text { D: } 51.1\end{array}$ & & $\begin{array}{l}\text { Four visits to the GP per } \\
\text { year } \\
\text { Blood glucose and weight } \\
\text { are measured at every } \\
\text { visit. Other blood } \\
\text { measures and feet and } \\
\text { eye inspection every year }\end{array}$ & $\begin{array}{l}\text { Computer-based } \\
\text { patient records }\end{array}$ & $\begin{array}{l}\text { Guideline } \\
\text { recommending four } \\
\text { visits to the GP per } \\
\text { year }\end{array}$ \\
\hline $\begin{array}{l}\text { Uitewaal et al. } \\
(2005)\end{array}$ & $\mathrm{NL}$ & $\mathrm{CT}$ & 12 & $\begin{array}{l}\text { I: } 53 \\
\text { C: } 51\end{array}$ & $\begin{array}{l}\text { I: } 50.6(9.3) \\
\text { C: } 53.5(6.2)\end{array}$ & $\begin{array}{l}\text { I: } 40 \\
\text { C: } 38\end{array}$ & $\begin{array}{l}\text { Culturally acceptable } \\
\text { and ethnic specific } \\
\text { diabetes program for } \\
\text { Turkish diabetes } \\
\text { patients, consisting of } \\
\text { seven individual } \\
\text { education sessions } \\
\text { and three group } \\
\text { sessions } \\
\text { Program was based on } \\
\text { three principles: peer } \\
\text { education, tailoring } \\
\text { and the Health } \\
\text { Education Model }\end{array}$ & $\begin{array}{l}\text { Individual sessions } \\
\text { consisting of four } \\
\text { sessions with the } \\
\text { educator and patient } \\
\text { together and three } \\
\text { 'triangle' sessions with } \\
\text { the GP, educator and } \\
\text { patient present, to discuss } \\
\text { three-monthly } \\
\text { assessment of glycemic } \\
\text { control and } \\
\text { cardiovascular risk factors } \\
\text { Patients were encouraged } \\
\text { to have one of the } \\
\text { individual sessions with } \\
\text { the dietician and one with } \\
\text { the partner present, } \\
\text { although this was not } \\
\text { obligatory }\end{array}$ & $\begin{array}{l}\text { Computer-based } \\
\text { patient records }\end{array}$ & \\
\hline
\end{tabular}


Table 2 (Continued)

\begin{tabular}{|c|c|c|c|c|c|c|c|c|c|c|}
\hline \multicolumn{4}{|l|}{ Study characteristics } & \multicolumn{3}{|c|}{ Sample characteristics } & \multicolumn{4}{|l|}{ CCM } \\
\hline \multirow[b]{2}{*}{ Study } & \multirow[b]{2}{*}{ Country } & \multirow[b]{2}{*}{$\begin{array}{l}\text { Study } \\
\text { design }\end{array}$} & \multirow[b]{2}{*}{$\begin{array}{l}\text { Follow- } \\
\text { up } \\
\text { (months) }\end{array}$} & \multirow[b]{2}{*}{$n$} & \multirow[b]{2}{*}{$\begin{array}{l}\text { Age (SD or } \\
\text { range) }\end{array}$} & \multirow[b]{2}{*}{$\begin{array}{l}\text { Sex } \\
\text { (\% male) }\end{array}$} & \multicolumn{4}{|c|}{ Description of components } \\
\hline & & & & & & & $\begin{array}{l}\text { Self-management } \\
\text { support }\end{array}$ & Delivery system design & $\begin{array}{l}\text { Clinical information } \\
\text { systems }\end{array}$ & Decision support \\
\hline $\begin{array}{l}\text { Whaba and Chang } \\
\text { (2007) }\end{array}$ & USA & CS & N/A & 136 & $59.7(15.2)$ & 51.5 & $\begin{array}{l}\text { Individual care plan } \\
\text { Self-monitoring of } \\
\text { blood glucose }\end{array}$ & $\begin{array}{l}\text { Care team (dietitian, DM } \\
\text { nurse educator and } \\
\text { physician) } \\
\text { Patient referred to } \\
\text { ophthalmologic and } \\
\text { podiatric evaluations as } \\
\text { soon as the diagnosis of } \\
\text { DM was made } \\
\text { Regular follow-up }\end{array}$ & $\begin{array}{l}\text { Patient prescribed a } \\
\text { glucose meter and } \\
\text { advised to keep a } \\
\text { diary of those } \\
\text { readings to share } \\
\text { with the physician at } \\
\text { each office visit }\end{array}$ & $\begin{array}{l}\text { Plan of care } \\
\text { developed } \\
\text { specifically for the } \\
\text { patient's clinical } \\
\text { condition } \\
\text { Laboratory tests were } \\
\text { conducted at least } \\
\text { twice a year } \\
\text { Compliance with diet } \\
\text { and medications was } \\
\text { assessed at each } \\
\text { visit } \\
\text { A DM flow sheet was } \\
\text { created for each } \\
\text { patient to keep track } \\
\text { of the laboratory } \\
\text { values, medications, } \\
\text { and immunizations }\end{array}$ \\
\hline
\end{tabular}

$\mathrm{CCM}=$ chronic care model; Jor = Jordan; $\mathrm{CS}=$ cross-sectional; $\mathrm{N} / \mathrm{A}=$ not applicable; $\mathrm{RC}=$ retrospective cohort; $\mathrm{ES}=\mathrm{Spain} ; \mathrm{PC}=$ prospective cohort; $\mathrm{DK}=\mathrm{Denmark} ; \mathrm{NL}=$ the Netherlands; Mex = Mexico; $\mathrm{RCT}=$ randomized controlled trials; $\mathrm{AUS}=$ Australia; $\mathrm{CH}=\mathrm{China}$; $\mathrm{SWE}=\mathrm{Sweden} ; \mathrm{HbA} 1 \mathrm{c}=$ hemoglobin $\mathrm{A} 1 \mathrm{c}$; $\mathrm{SG}=\mathrm{Singapore}$; $\mathrm{TH}=\mathrm{Thailand}$; $\mathrm{CT}=$ controlled trial; $\mathrm{PCP}=$ prospective cohort physician; $\mathrm{T}=$ Turkish; $\mathrm{D}=$ Dutch; $\mathrm{DM}=$ diabetes mellitus 
Table 3 Subgroup intervention effects on hemoglobin A1c (HbA1c)

Study

Uitewaal et al. (2005) ${ }^{c}$ Baseline HbA1c, sex, age, DM duration, DM medication,

Moreira et al. (2015) N/A

Quinn et al. (2016) Study group, time, age, all two-way interactions and three-way interaction

$\begin{array}{ll}\text { Nielsen et al. (2006) } & \begin{array}{c}\text { Clustering effect at the general practitioner level, interaction } \\ \text { between age and baseline HbA1c, DM duration, BMI, number } \\ \text { DM-related consultations, interaction between the patients' } \\ \text { physical activity level, antidiabetic medication and dietary }\end{array}\end{array}$

Nielsen et al. (2006) $\begin{aligned} & \text { Clustering effect at the general practitioner level, interaction } \\ & \text { between age and baseline HbA1c, DM duration, BMI, number of } \\ & \text { DM-related consultations, interaction between the patients' } \\ & \text { physical activity level, antidiabetic medication and dietary }\end{aligned}$

Nielsen et al. (2006) $\begin{aligned} & \text { Clustering effect at the general practitioner level, interaction } \\ & \text { between age and baseline HbA1c, DM duration, BMI, number } \\ & \text { DM-related consultations, interaction between the patients' } \\ & \text { physical activity level, antidiabetic medication and dietary }\end{aligned}$

Nielsen et al. (2006) Clustering effect at the general practitioner level, interaction
between age and baseline HbA1c, DM duration, BMI, number
DM-related consultations, interaction between the patient
physical activity level, antidiabetic medication and dietary

Rothman et al. (2004) Baseline HbA1c, age, race, sex, income, DM medication, DM duration, income

Moreira et al. (2015) N/A

Weak

\begin{tabular}{cccccc}
\hline \multicolumn{7}{c}{ Context-related characteristics } \\
\hline Low & High & Monthly & Monthly & $\leqslant$ Four & $>$ Four \\
literacy & literacy & income & income & years of & years of \\
status & status & $\leqslant \$ 1826$ & $>\$ 11826$ & schooling & schooling \\
\hline
\end{tabular}

\begin{tabular}{|c|c|c|c|c|c|}
\hline \multicolumn{6}{|c|}{ Health-related characteristics } \\
\hline $\begin{array}{c}\mathrm{FBG} \\
>10 \mathrm{mmol} / \\
\mathrm{L}\end{array}$ & $\begin{array}{c}\text { FBG } \\
\leqslant 10 \mathrm{mmol} / \\
\mathrm{L}\end{array}$ & $\begin{array}{c}\text { Depression } \\
\text { Yes }\end{array}$ & $\begin{array}{l}\text { Depression } \\
\text { No }\end{array}$ & $\begin{array}{c}\text { DM } \\
\text { duration } \\
<\text { five } \\
\text { years }\end{array}$ & $\begin{array}{c}\text { DM } \\
\text { duration } \\
\geqslant \text { five } \\
\text { years }\end{array}$ \\
\hline
\end{tabular}

Groeneveld et al. N/A

(2001)

Trief et al. (2006)

Baseline HbA1c, ethnicity, age, sex, marital status, years of education, DM duration, insulin use, smoking, co-morbidity,

Moreira et al. (2015)
Moderate

Global

quality

rating

Weak

Weak

Weak status 0

Weak

Weak

Person-related characteristics

Female Male Lower age ${ }^{a}$ Higher

age $^{\text {b }}$

Weak

Weak

$\mathrm{DM}=$ diabetes mellitus; $\mathrm{BMI}=$ body mass index; $\mathrm{N} / \mathrm{A}$ : not applicable; $\mathrm{FBG}=$ fasting blood glucose.

a Lower age: $\leqslant 52$ years (Moreira et al., 2015), $<55$ years (Quinn et al., 2016).

b Higher age: $>52$ years (Moreira et al., 2015), $\geqslant 55$ years (Quinn et al., 2016).

${ }^{c}$ Intervention and control groups only consisted of patients with a baseline $\mathrm{HbA} 1 \mathrm{c}>7 \%$.

$\mathrm{o}$ : No significant relationship between the characteristic with $\mathrm{HbA} 1 \mathrm{c}$ for people in the intervention group compared to usual care; - : significant negative relationship between the characteristic with $\mathrm{HbA1c}$ for patients in the intervention group compared to usual care. 
at follow-up compared to patients receiving usual care (Moreira et al., 2015).

No RCTs assessed context-related characteristics as potential effect modifiers of the relationship between integrated diabetes care programs and $\mathrm{HbA} 1 \mathrm{c}$.

Prospective and retrospective cohort studies: In total, 11 prospective and retrospective cohort studies measured the effects of integrated diabetes care programs on HbA1c (Tables 4 and 5). Three studies compared the change in HbA1c between levels of patient characteristics (Rothman et al., 2003; Sperl-Hillen and O'Connor, 2005; Elissen et al., 2012). The other eight studies compared HbA1c levels at follow-up between levels of patient characteristics (El-Kebbi et al., 2003; Benoit et al., 2005; Mold et al., 2008; De Fine Olivarius et al., 2009; Robinson et al., 2009; Kellow et al., 2011; Cardenas-Valladolid et al., 2012; LeBlanc et al., 2015).

Most examined person-related characteristics were age $(n=11)$ and sex $(n=9)$. In seven studies the effect of integrated diabetes care programs on HbA1c differed significantly across ranges of age: younger patients had higher $\mathrm{HbA} 1 \mathrm{c}$ levels at follow-up compared to older patients $(n=5)$ and experienced greater change from baseline in HbA1c $(n=2)$ (El-Kebbi et al., 2003; Benoit et al., 2005; Sperl-Hillen and O'Connor, 2005; Mold et al., 2008; Kellow et al., 2011; Elissen et al., 2012; LeBlanc et al., 2015). As to the latter, the direction of the measured change in HbA1c differed: one study found a significant improvement (SperlHillen and O'Connor, 2005) and the other a significant increase (Elissen et al., 2012) in HbA1c. Age was not a significant effect modifier in the other four studies (Rothman et al., 2003; De Fine Olivarius et al., 2009; Robinson et al., 2009; Cardenas-Valladolid et al., 2012). The effect of integrated care on HbA1c did not differ between men and women in eight studies (El-Kebbi et al., 2003; Rothman et al., 2003; Benoit et al., 2005; Sperl-Hillen and O'Connor, 2005; De Fine Olivarius et al., 2009; Robinson et al., 2009; Kellow et al., 2011; LeBlanc et al., 2015). In one study females had significantly higher HbA1c levels at follow-up compared to males (Cardenas-Valladolid et al., 2012).

Most examined health-related characteristics were medication use $(n=8)$, baseline HbA1c $(n=7)$ and duration of type 2 diabetes $(n=6)$. The effect of integrated diabetes care programs on HbA1c was different for people on insulin therapy. These patients had higher HbA1c levels at followup compared with patients on diet and/or oral therapy in five studies(El-Kebbi et al., 2003; Benoit et al., 2005; Mold et al., 2008; De Fine Olivarius et al., 2009; LeBlanc et al., 2015) and less desirable changes in HbA1c from baseline (Sperl-Hillen and O'Connor, 2005). In two studies the relationship between integrated diabetes care programs and HbA1c did not differ between types of medication (Rothman et al., 2003; Kellow et al., 2011). In the studies assessing baseline HbA1c, patients with higher baseline HbA1c levels had higher HbA1c levels at follow-up $(n=3)$ (El-Kebbi et al., 2003; Benoit et al., 2005; LeBlanc et al., 2015), but did have greater improvements in HbA1c from baseline $(n=3)$ (Rothman et al., 2003; Sperl-Hillen and O'Connor, 2005; Elissen et al., 2012) compared to patients with lower baseline HbA1C levels. In one study baseline HbA1c was not a significant effect modifier (Kellow et al., 2011). The effect of integrated diabetes care programs on HbA1c differed significantly across ranges of diabetes duration in five studies. Patients with longer diabetes duration had significantly higher HbA1c levels at follow-up compared to patients with shorter diabetes duration $(n=5)$ (El-Kebbi et al., 2003; Benoit et al., 2005; Mold et al., 2008; Elissen et al., 2012; LeBlanc et al., 2015). In one study a significant opposite effect was found (Rothman et al., 2003).

Health insurance status was assessed by four studies. It did not seem to significantly modify the observed effect of integrated care on HbA1c in three studies (Rothman et al., 2003; Benoit et al., 2005; Robinson et al., 2009). Patients with no health insurance coverage had less desirable changes in HbA1c than those with health insurance coverage (Sperl-Hillen and O'Connor, 2005). No other context-related characteristics were examined by the included studies.

Cross-sectional studies: In total, six crosssectional studies measured the modifying effect of patient characteristics on the relationship between integrated diabetes care programs and HbA1c (Tables 4 and 5).

Most examined person-related characteristics were age $(n=6)$, body mass index (BMI) $(n=6)$ and sex $(n=5)$. Four studies of integrated care programs found non-significant associations between age and HbA1c (Ostgren et al., 2002; 
Table 4 Relationship between hemoglobin A1c (HbA1c) and person-related and context-related characteristics

Cardenas-
Valladolid et al.

(2012)

De Fine Olivarius

Age, sex, BMI, HbA1c baseline, SBP, TC, urinary Moderate o 0

o

et al. (2009) albumin

Person-related characteristics

$\begin{array}{lll}\text { Socio-demographics } & \text { Lifestyle } & \begin{array}{l}\text { Context- } \\ \text { related }\end{array}\end{array}$

characteristic

Variables entered in multivariate

Global

quality

Age Sex ${ }^{a}$ Ethnicity Marital

Education status $^{b}$

Health

rating

Prospective cohort studies

Moderate $0+$

Retrospective cohort studies

Benoit et al. (2005) Sperl-Hillen and

O'Connor (2005)

A1c, time, age, TC, DM duration, Medication

Strong $-0 \quad 0^{c}$

$+0$

$\mathrm{O}^{\mathrm{c}}$

Age, sex, baseline HbA1c, DM medication,

variable (age, sex, specialty), diabetes

educator visits, pharmacy coverage

Elissen et al (2012) N/A

El-Kebbi et al.

Weak

$-$

Year of presentation, age, sex, ethnicity, BMI, Strong

$-0 o^{h}$

,

(2003)

no. of interval visits, follow-up duration

LeBlanc et al. Age, sex, DM duration, DM medication,

Strong

$-0$ Charlson co-morbidity index

Kellow, Savige and Age, sex, OGTT, HbA1c, TC, HDL, TG, LDL/HDL Moderate

Khalil (2011)

ratio, weight change, body weight

Mold et al (2008) N/A

Robinson et al. N/A

Moderate

0

Weak 0 o $0^{+}$

o

Age, sex, ethnicity, education, insurance, BMI, Moderate $0 \quad 0 \quad 0^{m}$

$\mathrm{HbA1c}$, DM medication, hypertension

medication, hypercholesterolemia

medication, recent diagnosis of DM, DM

duration

Al Omari et al.

(2009)

De Alba Garcia

et al. (2006)

\section{DM medication, DM duration}

Weak o o

Age, sex, marital status, education, BMI,

Weak $0 \quad 0$

smoking follow diet, glucose family history of

DM, DM duration, DM medication, SBP, DBP,

TC, TG 


\begin{tabular}{|c|c|c|c|c|c|c|c|c|c|c|}
\hline \multirow[b]{3}{*}{ Study } & \multirow[b]{3}{*}{$\begin{array}{l}\text { Variables entered in multivariate } \\
\text { regression model }\end{array}$} & \multirow[b]{3}{*}{$\begin{array}{l}\text { Global } \\
\text { quality } \\
\text { rating }\end{array}$} & \multicolumn{7}{|c|}{ Person-related characteristics } & \multirow{3}{*}{$\begin{array}{l}\text { Context- } \\
\text { related } \\
\text { characteristic } \\
\begin{array}{l}\text { Health } \\
\text { insurance }\end{array}\end{array}$} \\
\hline & & & \multicolumn{5}{|c|}{ Socio-demographics } & \multicolumn{2}{|c|}{ Lifestyle } & \\
\hline & & & Age & Sex ${ }^{a}$ & Ethnicity & $\begin{array}{l}\text { Marital } \\
\text { status }^{\mathrm{b}}\end{array}$ & Education & $\mathrm{BMI}$ & Smoking & \\
\hline $\begin{array}{l}\text { Ostgren et al. } \\
(2002)\end{array}$ & Age, sex, waist-hip ratio, TG, $\beta$-cell function & Weak & 0 & & & & & 0 & & \\
\hline Quah et al. (2013) & $\begin{array}{l}\text { Age, sex, ethnicity, marital status, occupation, } \\
\text { housing type, DM duration, DM medication, } \\
\text { compliance to medication, self-monitoring, } \\
\text { BMI }\end{array}$ & Moderate & - & o & $0^{s}$ & 0 & $o^{t}$ & 0 & $\mathrm{o}^{\mathrm{u}}$ & \\
\hline $\begin{array}{l}\text { Taweepolcharoen } \\
\text { et al. (2006) }\end{array}$ & $\begin{array}{l}\text { Age, sex, DM duration, BMI, BP, fasting } \\
\text { glucose, TG, HDL, LDL }\end{array}$ & Weak & 0 & + & & & & 0 & & \\
\hline $\begin{array}{l}\text { Whaba and Chang } \\
\text { (2007) }\end{array}$ & $\begin{array}{l}\text { Age, DM duration, BMI, DM medication, } \\
\text { hypertension, hyperlipidemia }\end{array}$ & Moderate & - & 0 & & & & 0 & & \\
\hline
\end{tabular}

$\mathrm{BMI}=$ body mass index; $\mathrm{DM}=$ diabetes mellitus; $\mathrm{SBP}=$ systolic blood pressure; $\mathrm{TC}=$ total cholesterol; $\mathrm{PC}=\mathrm{prospective}$ cohort; $\mathrm{N} / \mathrm{A}=\mathrm{not}$ applicable; $\mathrm{OGTT}=$ oral glucose tolerance test; $\mathrm{HDL}=$ high-density lipoprotein; $\mathrm{LDL}=$ low-density lipoprotein; $\mathrm{TG}=$ triglycerides; $\mathrm{BP}=$ blood pressure.

${ }^{\text {a }} 0=$ male, $1=$ female.

b $0=$ not married, $1=$ married

c $0=$ Hispanic, black and white, $1=$ Asian.

d $0=$ current smoker, $1=$ past smoker, $2=$ never smoker.

e 0 insured, $1=$ County Medical Services, $3=$ uninsured

f $0=$ pharmacy coverage, $1=$ no pharmacy coverage.

g $0=$ current smoker, $1=$ none smoker/previous smoker.

${ }^{\mathrm{h}} 0=$ others, $1=$ African American.

i $0=$ non-smoker, $1=$ current smoker.

j $0=$ white, 1 = black Caribbean/African.

${ }^{\mathrm{k}} 0=$ white, $1=$ Asian, $2=$ black, $3=$ other.

$0=$ insured, $1=$ uninsured

$\mathrm{m}_{0}=$ black $1=$ others

${ }^{\mathrm{n}} 0=$ less than high school, $1=$ high school or higher.

${ }^{\circ} 0=$ Medicaid or pharmacy assistance programs, $1=$ no Medicaid or pharmacy assistance program.

${ }^{\mathrm{p}} 0=$ current smoker, $1=$ past and none smoker.

${ }^{\mathrm{q}} 0=$ none, $1=$ incomplete primary, $2=$ completed primary, $3=$ primary

$\mathrm{r} 0=$ smoker, $1=$ none smoker.

${ }^{s} 0=$ Chinese, $1=$ Malay, $2=$ Indian, $3=$ others.

${ }^{\mathrm{t}} 0=$ no formal education, $1=$ formal education.

u $0=$ none smoker, 1 = past smoker, $2=$ current smoker.

+ : positive significant relationship; o- non-significant relationship; - : significant negative relationship. 
Table 5 Relationship between hemoglobin A1c (HbA1c) and health-related characteristics

Valladolid

(2012) et al. (2009)

Age, sex, $\mathrm{BMI}, \mathrm{HbA} 1 \mathrm{c}$ baseline, SBP, TC, urinary albumin

Moderate

Health-related characteristics

Benoit et al. (2005) A1c, time, age, TC, DM duration, Medication

Sperl-Hillen and O'Connor (2005)

Retrospective cohort studies

Elissen et al.

HbA1c SBP DBP TC HDL LDL TG

Provid

DM

Medication $^{\mathrm{a}}$ \# $\mathrm{Co}_{-}$

quality visits

morbidities

\section{Prospective cohort studies}

Moderate

(2012)

El-Kebbi et al.

(2003)

Kellow, Savige

and Khalil (2011)

LeBlanc et al.

(2015)

$0+0$

Mold et al. (2008) N/A

Robinson et al.

Rothman et al. Age, sex, ethnicity, education,

(2003)

Age, sex, baseline HbA1c, DM Weak +

medication, depression,

co-morbidities, PC physician

variable (age, sex, specialty), diabetes educator visits,

pharmacy coverage

N/A

Year of presentation, age, sex ethnicity, BMI, DM duration,

baseline $\mathrm{HbA} 1 \mathrm{c}$, DM medication,

no. of interval visits, follow-up

duration

Age, sex, OGTT, HbA1c, TC, HDL, Moderate o

TG, LDL/HDL ratio, weight

change, body weight

Age, sex, DM duration, DM

Strong

0

medication, Charlson CO-

Weak

Strong

morbidity index

N/A

\section{Moderate}

Weak

insurance, BMI, HbA1c, DM

Moderate +

$+$

0

ation, hypertension

medication

hypercholesterolemia

medication, recent diagnosis of

DM, DM duration 
Table 5 (Continued)

\begin{tabular}{|c|c|c|c|c|c|c|c|c|c|c|c|c|c|}
\hline \multirow[b]{2}{*}{ Study } & \multirow[b]{2}{*}{$\begin{array}{l}\text { Variables entered in multivariate } \\
\text { regression model }\end{array}$} & \multirow[b]{2}{*}{$\begin{array}{l}\text { Global } \\
\text { quality } \\
\text { rating }\end{array}$} & \multicolumn{11}{|c|}{ Health-related characteristics } \\
\hline & & & HbA1c & SBP & DBP & TC & HDL & LDL & TG & $\begin{array}{l}\# \\
\text { Providers } \\
\text { visits }\end{array}$ & $\begin{array}{l}\text { DM } \\
\text { duration }\end{array}$ & Medication $^{a}$ & $\begin{array}{l}\text { \# Co- } \\
\text { morbidities }\end{array}$ \\
\hline \multicolumn{14}{|c|}{ Cross-sectional studies } \\
\hline $\begin{array}{l}\text { Al Omari et al. } \\
(2009)\end{array}$ & DM medication, DM duration & Weak & & & & 0 & 0 & 0 & 0 & & + & + & \\
\hline $\begin{array}{l}\text { De Alba Garcia } \\
\text { et al. (2006) }\end{array}$ & $\begin{array}{l}\text { Age, sex, marital status, } \\
\text { education, BMI, smoking, follow } \\
\text { diet, glucose, family history of } \\
\text { DM, DM duration, DM } \\
\text { medication, SBP, DBP, TC, TG }\end{array}$ & Weak & & 0 & 0 & 0 & & & 0 & 0 & + & + & \\
\hline $\begin{array}{l}\text { Ostgren et al. } \\
(2002)\end{array}$ & $\begin{array}{l}\text { Age, sex, waist-hip ratio, TG, } \beta \text {-cell } \\
\text { function }\end{array}$ & Weak & & - & - & 0 & & & + & & 0 & & \\
\hline Quah et al. (2013) & $\begin{array}{l}\text { Age, sex, ethnicity, marital status, } \\
\text { occupation, housing type, DM } \\
\text { duration, DM medication, } \\
\text { compliance to medication, } \\
\text { self-monitoring, BMI }\end{array}$ & Moderate & & & & & & & & & + & + & 0 \\
\hline $\begin{array}{l}\text { Taweepolcharoen } \\
\text { et al. (2006) }\end{array}$ & $\begin{array}{l}\text { Age, sex, DM duration, BMI, BP, } \\
\text { fasting glucose, TG, HDL, LDL }\end{array}$ & Weak & & & & & 0 & 0 & 0 & & + & & \\
\hline $\begin{array}{l}\text { Whaba and Chang } \\
\text { (2007) }\end{array}$ & $\begin{array}{l}\text { Age, DM duration, BMI, DM } \\
\text { medication, hypertension, } \\
\text { hyperlipidemia }\end{array}$ & Moderate & & & & & & & & & 0 & 0 & 0 \\
\hline
\end{tabular}

$\mathrm{SBP}=$ systolic blood pressure; $\mathrm{DBP}=$ diastolic blood pressure; $\mathrm{TC}=$ total cholesterol; $\mathrm{HDL}=$ high-density lipoprotein; $\mathrm{LDL}=$ low-density lipoprotein; $\mathrm{TG}=$ triglycerides; $\mathrm{DM}=$ diabetes mellitus; $\mathrm{PC}=$ primary care; $\mathrm{OGTT}=$ oral glucose tolerance test; $\mathrm{N} / \mathrm{A}=\mathrm{not}$ applicable; $\mathrm{BMI}=$ body $\mathrm{mass}$ index; $\mathrm{BP}=$ blood pressure.

+: positive significant relationship; o: non-significant relationship; - : significant negative relationship.

$0=$ no insulin, $1=$ insulin 
De Alba Garcia et al., 2006; Taweepolcharoen et al., 2006; Al Omari et al., 2009). In two studies significant associations were found: in these studies, younger patients had higher HbA1c levels (Wahba and Chang, 2007; Quah et al., 2013). The effect of integrated diabetes care programs on $\mathrm{HbA} 1 \mathrm{c}$ did not significantly differ between levels of BMI in all studies (Ostgren et al., 2002; De Alba Garcia et al., 2006; Taweepolcharoen et al., 2006; Wahba and Chang, 2007; Al Omari et al., 2009; Quah et al., 2013). The effect on HbA1c did also not differ between men and women in four studies (De Alba Garcia et al., 2006; Wahba and Chang, 2007; Al Omari et al., 2009; Quah et al., 2013). In one study females had significantly higher HbA1c levels compared to males (Taweepolcharoen et al., 2006).

Most examined health-related characteristics were duration of type 2 diabetes $(n=6)$ and medication use $(n=4)$. The effect of integrated care programs on HbA1c differed significantly across ranges of diabetes duration in four studies (De Alba Garcia et al., 2006; Taweepolcharoen et al., 2006; Al Omari et al., 2009; Quah et al., 2013). Patients with longer diabetes duration had higher HbA1c levels compared to patients with shorter diabetes duration in these studies. In two studies diabetes duration was not a significant effect modifier (Ostgren et al., 2002; Wahba and Chang, 2007). The effect of integrated care programs on HbA1c was also different for people on insulin therapy. These patients had higher HbA1c concentrations compared with patients on diet and/or oral therapy in three studies (De Alba Garcia et al., 2006; Al Omari et al., 2009; Quah et al., 2013). In one study type of medication was not a significant effect modifier (Wahba and Chang, 2007).

No context-related characteristics were assessed by three or more studies.

\section{$L D L-c$}

Three prospective and retrospective cohort studies measured the effect of integrated diabetes care programs on LDL-c. The RCTs and crosssectional studies included in this review did not measure this effect. In total, 11 patient characteristics were assessed by the studies. Only those results that were assessed by at least two studies will be discussed.

Prospective and retrospective cohort studies: The person-related characteristic age was examined by three studies (Sperl-Hillen and O'Connor, 2005; Robinson et al., 2009; Elissen et al., 2012). The relationship between age and LDL-c was inconsistent: a negative and positive as well as a non-significant relationship were found.

The modifying effect of baseline LDL-c on the relationship between integrated diabetes care programs and changes in LDL-c over baseline was assessed by two studies (Sperl-Hillen and O'Connor, 2005; Elissen et al., 2012). Both found that patients with higher baseline LDL-c had greater LDL-c improvements.

No context-related characteristics were assessed by the included studies.

\section{$S B P$}

Four retrospective and prospective cohort studies measured the effect of integrated diabetes care programs on SBP. In total, nine patient characteristics were assessed by the studies. Only those results that were assessed by at least two studies will be discussed.

Retrospective cohort and prospective cohort studies: Age was measured by three studies (Mold et al., 2008; Robinson et al., 2009; Elissen et al., 2012). These studies found that higher age was associated with higher SBP at follow-up (Mold et al., 2008; Robinson et al., 2009) and greater improvement (Elissen et al., 2012). The modifying effect of ethnicity on integrated care programs and SBP was measured by two studies (Mold et al., 2008; Robinson et al., 2009). The effect was unclear, as results were inconsistent between these studies. Four other characteristics were assessed, one context-related and three health-related characteristics, by one study each.

\section{Health-care utilization}

Health-care utilization was assessed by three studies: one RCT (Nielsen et al., 2006), one retrospective cohort study (Uitewaal et al., 2004) and one cross-sectional study (Liu et al., 2013). Together they measured the modifying effect of integrated care programs and health-care utilization for five person-related characteristics, one context-related characteristic and one healthrelated characteristic. Most examined characteristic was sex, which was measured by two studies (Nielsen et al., 2006; Liu et al., 2013). Nielsen et al. (2006) 
found that females in the intervention group had statistically significant more GP consultations per year compared to females in the control group (Nielsen et al., 2006). For males, no difference was found. Liu et al. found that the effect of integrated diabetes care programs on health-care utilization was different between males and females (Liu et al., 2013). Females had higher utilization of community health centers compared to male.

\section{Discussion}

This paper presents a literature review on relevant patient characteristics for guiding tailored integrated type 2 diabetes care in primary care. HbA1c was considered an outcome in $93 \%$ of the 27 studies identified. Many different patient characteristics were investigated by these studies. Findings indicate that the effect of integrated primary care programs on HbA1c differs significantly according to a number of person and health-related characteristics. Younger age, longer disease duration, higher baseline $\mathrm{HbA} 1 \mathrm{c}$ and insulin therapy were associated with higher HbA1c levels. Health insurance status, living situation and income were the only context-related characteristics in the included studies and were not frequently assessed.

Compared to HbA1c, LDL-c, SBP and healthcare utilization were included far less. It was found that higher baseline LDL-c lead to greater LDL-c improvement. Patients with higher age had higher SBP levels at follow-up as well as greater improvements in SBP compared to younger patients. The relationship between integrated care and health-care utilization seemed to be modified by sex: women had more consultations per year compared to men.

Several factors might explain the elevated HbA1c levels in a subset of patients with type 2 diabetes. Younger patients tend be more non-adherent to oral medication therapy and experience less profound diabetes-related health problems than older patients (Pyatak et al., 2014; Tunceli et al., 2015). The latter might cause them to believe that a proactive attitude toward their disease is less important. Moreover, younger patients and/or those with longer disease duration undergo a more rapid decline in $\beta$ cell function and pancreatic insulin secretion, resulting in the need for a more complex and intensive drug therapy (Al Omari et al., 2009; Fonseca, 2009; Khattab et al., 2010; Kellow et al., 2011). Higher HbA1c levels for patients on insulin therapy compared to patients on diet and/or oral therapy could be due to a delayed start or low intensity of insulin therapy (Abraira et al., 1995; El-Kebbi et al., 2003; Mosenzon and Raz, 2013). Furthermore, maintaining glycemic control, while minimizing hypoglycemia and sticking to a diet might be difficult (Jin et al., 2008; Quah et al., 2013).

High HbA1c at baseline also seemed to be predictive of later HbA1c. First, type 2 diabetes is a heterogeneous disease in both pathogenesis and clinical manifestation (Inzucchi et al., 2012), thus a high HbA1c at baseline and at follow-up could be due to decreased insulin sensitivity, secretion and $\beta$-cell dysfunction (Heianza et al., 2012). Second, unhealthy lifestyle habits, such as low physical activity, and a diet rich in carbohydrates have been associated with less glycemic control (Mozaffarian et al., 2009; Inzucchi et al., 2012). Changing these lifestyle factors is easier said than done, making it difficult for patients to improve their glycemic control.

Several factors could explain the differences in levels of LDL-c, SBP and health-care utilization between levels of patient characteristics. Prescription of statins usually follows when LDL-c level is $2.5 \mathrm{mmol} / \mathrm{L}$ or higher, possibly leading to greater improvements in LDL-c for those patients with high baseline LDL-c levels (The Dutch college of general practitioners, 2011). The higher SBP levels at follow-up for older patients may be due to less stringent treatment targets (van Hateren et al., 2012; James et al., 2014). The greater health-care utilization by women compared to men might be explained by the difference in perception of illness between men and women. According to some studies, it is more culturally and socially accepted for women to be ill than it is for men (De Visser et al., 2009).

Overall, our results indicate the need to implement integrated diabetes care programs specifically tailored to the needs, values and preferences of younger patients and to those on insulin therapy, with longer disease duration and/or higher HbA1c levels and older patients with high SBP levels. These effect modifiers can help to provide the right care to the right person at the right time. At this moment, not every patient with 
these characteristics receives such care. Current practice might therefore not be suitable for all patients. Lack of motivation, family support and feeling burned-out from managing diabetes are reported barriers to optimal self-management (Browne et al., 2013). To tackle these barriers, diabetes treatment programs should take them into account by, for example, providing shared decision making and simple and specific instructions and advice, involving family members and offering online consultations or evening primary care opening hours. In addition to patients who find it difficult to keep their diabetes under control, there is a large group of patients who does manage to control their diabetes (Rothe et al., 2008; Elissen et al., 2012). For these patients, fewer visits to primary care might have similar outcomes and thus should be taken into consideration by both the GP and the patient. Allowing care givers to provide care based on patient characteristics constitutes a promising approach for achieving the so-called 'Triple Aim' by: (1) improving patient experience, by including patients' care needs, preferences, and abilities in treatment decisions; (2) improving population health and quality of life, by supporting tailored diabetes care; and (3) reducing the per capita cost of diabetes care, by reducing the over-, under- and misuse of health-care services (Berwick et al., 2008).

This review has several limitations that should be taken into account. First, given the scarceness of studies assessing the differences in the effect of integrated diabetes care programs on diabetes control measures by levels of patient characteristics, it was decided to include RCTs, prospective and retrospective cohort studies. However, this introduced significant heterogeneity and made it impossible to conduct a meta-analysis. Second, quality of the studies was weak for most studies. This was mainly due to the cross-sectional study design of more than one-third of the studies and the use of less robust statistical methods. Fortunately, it is unlikely that these studies altered the results, as their findings were similar to those of the other, more robust studies. Third, very few contextand person-related characteristics were analyzed. Studies performed in a non-integrated diabetes care setting, found that context-related characteristics, such as socio-economic status and social network, are associated with measures of diabetes control and are likely to be strong predictors of diabetes control (Jotkowitz et al., 2006; Nam et al., 2011). Person-related characteristics, such as low mastery and low self-efficacy, have been related to negative health outcomes (Bosma et al., 2014; Elissen et al., 2017). Traditionally, researchers and care providers have looked at diabetes from a mostly biomedical viewpoint, which might explain the relatively scarce collection of context- and person-related characteristics in routinely collected individual patient data (Hasnain-Wynia and Baker, 2006).

The current review provides a good understanding of which characteristics can help to identify patients with different health-care needs and preferences. However, to implement an effective integrated type 2 diabetes tailored care program, it is necessary to know which context- and personrelated characteristics are important to identify patients. Furthermore, implementation of an effective tailored diabetes care program is only possible by taking into account the care preferences of patients and caregivers. In the next phase of the PROFILe project (Elissen et al., 2016), data rich in non-health-related characteristics will be analyzed to assess which of these are predictors of diabetes control measures and a discrete choice experiment will be conducted to gain knowledge on patients' care preferences as a first step toward patient-centered diabetes care.

\section{Acknowledgments}

None.

\section{Financial Support}

This PROFILe project was supported by a grant from Novo Nordisk Netherlands (no grant number). The sponsor had no role in study design, in the collection, analysis and interpretation of the data; in the writing of the report; and in the decision to submit the article for publication.

\section{Conflicts of interest}

None.

\section{Supplementary material}

To view supplementary material for this article, please visit https://doi.org/10.1017/S14634236 1800004X 


\section{References}

Abraira, C., Colwell, J.A., Nuttall, F.Q., Sawin, C.T., Nagel, N.J., Comstock, J.P., Emanuele, N.V., Levin, S.R., Henderson, W. and Lee, H.S. 1995: Veterans Affairs Cooperative Study on glycemic control and complications in type II diabetes (VA CSDM). Results of the feasibility trial. Veterans Affairs Cooperative Study in Type II Diabetes. Diabetes Care 18, 1113-123.

Al Omari, M., Khader, Y., Dauod, A.S., Al-Akour, N., Khassawneh, A.H., Al-Ashker, E. and Al-Shdifat, A. 2009: Glycaemic control among patients with type 2 diabetes mellitus treated in primary care setting in Jordan. Primary Care Diabetes 3, 173-79.

American geriatrics society expert panel on person-centered care. 2016: Person-centered care: a definition and essential elements. Journal of the American Geriatrics Society 64, 15-18.

Andersen, R. and Newman, J.F. 1973: Societal and individual determinants of medical care utilization in the United States. Milbank Memorial Fund Quarterly. Health and Society 51, 95-124.

Armijo-Olivo, S., Stiles, C.R., Hagen, N.A., Biondo, P.D. and Cummings, G.G. 2012: Assessment of study quality for systematic reviews: a comparison of the Cochrane Collaboration Risk of Bias Tool and the Effective Public Health Practice Project Quality Assessment Tool: methodological research. Journal of Evaluation in Clinical Practice 18, $12-18$.

Austin, B., Wagner, E., Hindmarsh, M. and Davis, C. 2000: Elements of effective chronic care: a model for optimizing outcomes for the chronically ill. Epilepsy and Behavior 1, S15-20.

Benoit, S.R., Fleming, R., Philis-Tsimikas, A. and Ji, M. 2005: Predictors of glycemic control among patients with Type 2 diabetes: a longitudinal study. BMC Public Health 5, 36.

Berwick, D.M., Nolan, T.W. and Whittington, J. 2008: The triple aim: care, health, and cost. Health Affairs (Millwood) 27, 759-69.

Bosma, H., Theunissen, M.J., Verdonk, P. and Feron, F. 2014: Low control beliefs in relation to school dropout and poor health: findings from the SIODO case-control study. BMC Public Health 14, 1237.

Browne, J.L., Scibilia, R. and Speight, J. 2013: The needs, concerns, and characteristics of younger Australian adults with Type 2 diabetes. Diabetic Medicine 30, 620-26.

Busetto, L., Luijkx, K.G., Elissen, A.M. and Vrijhoef, H.J. 2016: Intervention types and outcomes of integrated care for diabetes mellitus type 2: a systematic review. Journal of Evaluation in Clinical Practice 22, 299-310.

Cardenas-Valladolid, J., Salinero-Fort, M.A., Gomez-Campelo, P., De Burgos-Lunar, C., Abanades-Herranz, J.C., Arnal-Selfa, R. and Andres, A.L. 2012: Effectiveness of standardized Nursing Care Plans in health outcomes in patients with type 2 Diabetes Mellitus: a two-year prospective follow-up study. PLoS One 7, e43870.
Commitee on Quality of Health Care in America; Institute of Medicine. 2001. Improving the 21st-century health care system crossing the quality chasm: a new health system for the 21st century. Washington, DC: National Academy Press.

Constand, M.K., MacDermid, J.C., Dal Bello-Haas, V. and Law, M. 2014: Scoping review of patient-centered care approaches in healthcare. BMC Health Services Research 14, 271.

Coulourides Kogan, A., Wilber, K. and Mosqueda, L. 2016: Moving toward implementation of person-centered care for older adults in community-based medical and social service settings: "you only get things done when working in concert with clients". Journal of the American Geriatrics Society 64, e8-14.

Coulter, A., Entwistle, V.A., Eccles, A., Ryan, S., Shepperd, S. and Perera, R. 2015: Personalised care planning for adults with chronic or long-term health conditions. Cochrane Database of Systematic Reviews 3, CD010523.

De Alba Garcia, J.E.G., Dallo, F.J., Salcedo Rocha, A.L., Rodriguez, C.C., Perez, N., Baer, R.D. and Weller, S.C. 2006: The relative effect of self-management practices on glycaemic control in type 2 diabetic patients in Mexico. Chronic Illness 2, 77-85.

De Fine Olivarius, N., Siersma, V., Hansen, L.J. Drivsholm, T. and Horder, M. 2009: Changes in levels of haemoglobin A1c during the first 6 years after diagnosis of clinical type 2 diabetes. Scandinavian Journal of Clinical and Laboratory Investigation 69, 851-57.

De Visser, R.O., Smith, J.A. and McDonnell, E.J. 2009: 'That's not masculine': masculine capital and health-related behaviour. Journal of Health Psychology 14, 1047-58.

The Dutch College of General Practitioners. 2011. Multidisciplinary practice guideline cardiovascular risk management. Houten: Bohn Stafleu van Loghum.

Elissen, A.M., Hertroijs, D.F., Schaper, N.C., Vrijhoef, H.J. and Ruwaard, D. 2016: Profiling patients' healthcare needs to support integrated, person-centered models for long-term disease management (profile): research design. International Journal of Integrated Care 16, 1.

Elissen, A.M.J., Duimel-Peeters, I.G.P., Spreeuwenberg, C., Spreeuwenberg, M. and Vrijhoef, H.J.M. 2012: Toward tailored disease management for type 2 diabetes. American Journal of Managed Care 18, 619-30.

Elissen, A.M.J., Hertroijs, D.F.L., Schaper, N.C., Bosma, H., Dagnelie, P.C., Henry, R.M., Van Der Kallen, C.J., Koster, A., Schram, M.T., Stehouwer, C.D.A., Schouten, J., Berendschot, T. and Ruwaard, D. 2017: Differences in biopsychosocial profiles of diabetes patients by level of glycaemic control and health-related quality of life: the Maastricht Study. PLoS One 12, e0182053.

El-Kebbi, I.M., Cook, C.B., Ziemer, D.C., Miller, C.D., Gallina, D.L. and Phillips, L.S. 2003: Association of younger age with poor glycemic control and obesity in urban African Americans with type 2 diabetes. Archives of Internal Medicine 163, 69-75. 
Epstein, R.M. and Street, R.L. Jr. 2011: The values and value of patient-centered care. Annals of Family Medicine 9, 100-3.

Fonseca, V.A. 2009: Defining and characterizing the progression of type 2 diabetes. Diabetes Care 32 (Suppl 2), S151-156.

Goldberger, J.J. and Buxton, A.E. 2013: Personalized medicine vs guideline-based medicine. JAMA 309, 2559-560.

Groeneveld, Y., Petri, H., Hermans, J. and Springer, M. 2001: An assessment of structured care assistance in the management of patients with type 2 diabetes in general practice. Scandinavian Journal of Primary Health Care 19, 25-30.

Hasnain-Wynia, R. and Baker, D.W. 2006: Obtaining data on patient race, ethnicity, and primary language in health care organizations: current challenges and proposed solutions. Health Services Research 41, 1501-518.

Heianza, Y., Arase, Y., Fujihara, K., Tsuji, H., Saito, K., Hsieh, S.D., Kodama, S., Shimano, H., Yamada, N., Hara, S. and Sone, H. 2012: High normal HbA(1c) levels were associated with impaired insulin secretion without escalating insulin resistance in Japanese individuals: the Toranomon Hospital Health Management Center Study 8 (TOPICS 8). Diabetic Medicine 29, 1285-290.

International Diabetes Federation. 2015: IDF Diabetes Atlas, 7th Edition. Brussels: International Diabetes Federation.

Inzucchi, S.E., Bergenstal, R.M., Buse, J.B., Diamant, M., Ferrannini, E., Nauck, M., Peters, A.L., Tsapas, A., Wender, R., Matthews, D.R., American Diabetes, A. and European Association For The Study Of D. 2012: Management of hyperglycemia in type 2 diabetes: a patient-centered approach: position statement of the American Diabetes Association (ADA) and the European Association for the Study of Diabetes (EASD). Diabetes Care 35, 1364-379.

James, P.A., Oparil, S., Carter, B.L., Cushman, W.C., Dennison-Himmelfarb, C., Handler, J., Lackland, D.T., Lefevre, M.L., Mackenzie, T.D., Ogedegbe, O., Smith, S.C. Jr., Svetkey, L.P., Taler, S.J., Townsend, R.R., Wright, J.T. Jr., Narva, A.S. and Ortiz, E. 2014: 2014 Evidence-based guideline for the management of high blood pressure in adults: report from the panel members appointed to the Eighth Joint National Committee (JNC 8). JAMA 311, 507-20.

Jin, J., Sklar, G.E., Min Sen Oh, V. and Chuen Li, S. 2008: Factors affecting therapeutic compliance: a review from the patient's perspective. Therapeutics and Clinical Risk Management 4, 269-86.

Jotkowitz, A.B., Rabinowitz, G., Raskin Segal, A., Weitzman, R., Epstein, L. and Porath, A. 2006: Do patients with diabetes and low socioeconomic status receive less care and have worse outcomes? A national study. American Journal of Medicine 119, 665-69.

Kellow, N.J., Savige, G.S. and Khalil, H. 2011: Predictors of poor glycaemic control during the initial five years postdiagnosis in rural adults with type 2 diabetes. Australian Journal of Rural Health 19, 267-74.

Khattab, M., Khader, Y.S., Al-Khawaldeh, A. and Ajlouni, K. 2010: Factors associated with poor glycemic control among patients with type 2 diabetes. Journal of Diabetes and Its Complications 24, 84-89.

Kodner, D.L. 2009: All together now: a conceptual exploration of integrated care. Healthcare Quarterly 13, 6-15.

LeBlanc, E.S., Rosales, A.G., Kachroo, S., Mukherjee, J., Funk, K.L. and Nichols, G.A. 2015: Do patient or provider characteristics impact management of diabetes? American Journal of Managed Care 21, 597-606.

Liu, L.J., Li, Y., Sha, K., Wang, Y. and He, X. 2013: Patient assessment of chronic illness care, glycemic control and the utilization of community health care among the patients with type 2 diabetes in Shanghai, China. PLoS One 8, e73010.

Luijks, H.D., De Grauw, W.J., Bor, J.H., Van Weel, C., LagroJanssen, A.L., Biermans, M.C. and Schermer, T.R. 2015: Exploring the impact of chronic obstructive pulmonary disease (COPD) on diabetes control in diabetes patients: a prospective observational study in general practice. NPJ Primary Care Respiratory Medicine 25, (no pagination).

McCulloch, D.K., Price, M.J., Hindmarsh, M. and Wagner, E. H. 1998: A population-based approach to diabetes management in a primary care setting: early results and lessons learned. Effective Clinical Practice 1, 12-22.

Moher, D., Liberati, A., Tetzlaff, J., Altman, D.G. and Group, P. 2009: Preferred reporting items for systematic reviews and meta-analyses: the PRISMA Statement. Open Medicine 3, e123-130.

Mold, F., While, A. and Forbes, A. 2008: The management of type 2 diabetes care: the challenge within primary care. Practical Diabetes International 25, 28-36.

Moreira, R.C., Mantovani Mde, F. and Soriano, J.V. 2015: Nursing case management and glycemic control among Brazilians with type 2 diabetes: pragmatic clinical trial. Nursing Research 64, 272-81.

Mosenzon, O. and Raz, I. 2013: Intensification of insulin therapy for type 2 diabetic patients in primary care: basal-bolus regimen versus premix insulin analogs: when and for whom? Diabetes Care 36, S212-218.

Mozaffarian, D., Kamineni, A., Carnethon, M., Djousse, L., Mukamal, K.J. and Siscovick, D. 2009: Lifestyle risk factors and new-onset diabetes mellitus in older adults: the cardiovascular health study. Archives of Internal Medicine 169, 798-807.

Nam, S., Chesla, C., Stotts, N.A., Kroon, L. and Janson, S.L. 2011: Barriers to diabetes management: patient and provider factors. Diabetes Research and Clinical Practice 93, 1-9.

Nielsen, A.B.S., de Fine Olivarius, N., Gannik, D., Hindsberger, C. and Hollnagel, H. 2006: Structured personal diabetes care in primary health care affects only women's HbA1c. Diabetes Care 29, 963-69.

Ostgren, C.J., Lindblad, U., Ranstam, J., Melander, A. and Rastam, L. 2002: Glycaemic control, disease duration and beta-cell function in patients with Type 2 diabetes in a Swedish community. Skaraborg Hypertension and Diabetes Project. Diabetes Medicine 19, 125-29. 
Pimouguet, C., Le Goff, M., Thiebaut, R., Dartigues, J.F. and Helmer, C. 2011: Effectiveness of disease-management programs for improving diabetes care: a meta-analysis. CMAJ 183, E115-127.

Pyatak, E.A., Florindez, D., Peters, A.L. and Weigensberg, M.J. 2014: 'We are all gonna get diabetic these days': the impact of a living legacy of type 2 diabetes on Hispanic young adults' diabetes care. Diabetes Educator 40, 648-58.

Quah, J.H.M., Liu, Y.P., Luo, N., How, C.H. and Tay, E.G. 2013: Younger adult type 2 diabetic patients have poorer glycaemic control: a cross-sectional study in a primary care setting in Singapore. BMC Endocrine Disorders 13, 13-18.

Quinn, C.C., Shardell, M.D., Terrin, M.L., Barr, E.A., Park, D., Shaikh, F., Guralnik, J.M. and Gruber-Baldini, A.L. 2016: Mobile diabetes intervention for glycemic control in 45- to 64-year-old persons with type 2 diabetes. Journal of Applied Gerontology 35, 227-43.

Riddle, M.C. and Karl, D.M. 2012: Individualizing targets and tactics for high-risk patients with type 2 diabetes: practical lessons from ACCORD and other cardiovascular trials. Diabetes Care 35, 2100-107.

Robinson, S., Baron, R.B., Cooper, B. and Janson, S. 2009: Does health service use in a diabetes management program contribute to health disparities at a facility level? Optimizing resources with demographic predictors. Population Health Management 12, 139-47.

Rothe, U., Muller, G., Schwarz, P.E., Seifert, M., Kunath, H., Koch, R., Bergmann, S., Julius, U., Bornstein, S.R., Hanefeld, M. and Schulze, J. 2008: Evaluation of a diabetes management system based on practice guidelines, integrated care, and continuous quality management in a Federal State of Germany: a population-based approach to health care research. Diabetes Care 31, 863-68.

Rothman, R., Malone, R., Bryant, B., Horlen, C. and Pignone, M. 2003: Pharmacist-led, primary care-based disease management improves hemoglobin A1c in high-risk patients with diabetes. American Journal of Medical Quality 18, 51-58.

Rothman, R.L., DeWalt, D.A., Malone, R., Bryant, B., Shintani, A., Crigler, B., Weinberger, M. and Pignone, M. 2004: Influence of patient literacy on the effectiveness of a primary care-based diabetes disease management program. JAMA 292, 1711-716.

Scheen, A.J. 2016: Precision medicine: the future in diabetes care? Diabetes Research and Clinical Practice 117, 12-21.
Shaw, S., Rosen, R. and Rumbold, B. 2011. What is integrated care? London: Nuffield Trust.

Sperl-Hillen, J.M. and O'Connor, P.J. 2005: Factors driving diabetes care improvement in a large medical group: ten years of progress. American Journal of Managed Care 11, S177-185.

Tamayo, T., Rosenbauer, J., Wild, S.H., Spijkerman, A.M., Baan, C., Forouhi, N.G., Herder, C. and Rathmann, W. 2014: Diabetes in Europe: an update. Diabetes Research and Clinical Practice 103, 206-17.

Taweepolcharoen, N., Sumrithe, S., Kunentrasai, N., Phraisuwanna, S. 2006: Diabetes control in a university primary care setting in Thailand. Quality in Primary Care 14, 219-23.

Trief, P.M., Morin, P.C., Izquierdo, R., Teresi, J., Eimicke, J.P., Goland, R., Starren, J., Shea, S. and Weinstock, R.S. 2006: Depression and glycemic control in elderly ethnically diverse patients with diabetes: the IDEATel project. Diabetes Care 29, 830-35.

Tseng, M.M. and Hu, S.J. 2014: Mass customization. Manufacturing Technology. 836-43.

Tunceli, K., Iglay, K., Zhao, C., Brodovicz, K.G. and Radican, L. 2015: Factors associated with adherence to oral antihyperglycemic monotherapy in patients with type 2 diabetes mellitus in the United Kingdom. Diabetes Research and Clinical Practice 109, e27-31.

Uitewaal, P.J., Bruijnzeels, M.A., Bernsen, R.M., Voorham, A.J., Hoes, A.W. and Thomas, S. 2004: Diabetes care in Dutch general practice: differences between Turkish immigrants and Dutch patients. European Journal of Public Health 14, 15-18.

Uitewaal, P.J., Voorham, A.J., Bruijnzeels, M.A., Berghout, A., Bernsen, R.M., Trienekens, P.H., Hoes, A.W. and Thomas, S. 2005: No clear effect of diabetes education on glycaemic control for Turkish type 2 diabetes patients: a controlled experiment in general practice. Netherlands Journal of Medicine 63, 428-34.

van Hateren, K.J., Landman, G.W., Kleefstra, N., Houweling, S.T., Van Der Meer, K. and Bilo, H.J. 2012: Time for considering other blood pressure target values in elderly patients with type 2 diabetes? International Journal of Clinical Practice 66, 125-27.

Wahba, H. and Chang, Y.F. 2007: Factors associated with glycemic control in patients with type 2 diabetes mellitus in rural areas of the United States. Insulin 2, 134-41. 